\title{
2 Bewerten
}

Das folgende Kapitel dient zunächst der Diskussion linguistischer Ansätze und Modelle zur Beschreibung von Bewertungen im Allgemeinen. Hierbei liegt der Fokus zum einen auf handlungstheoretischen Ansätzen aus der Pragmatik der 1980er Jahre, die sich primär auf der Basis schriftlicher, teilweise fiktiver Texte mit dem Bewerten auf einer allgemeinen Ebene beschäftigen. Sie umfassen sowohl terminologische Eingrenzungen des Bewertungsbegriffs als auch Bewertungsmodelle, welche als Ergebnisse aus der Analyse von vor allem schriftlichen Texten hervorgegangen sind. ${ }^{2}$ Diese handlungstheoretischen Ansätze, die Bewerten als bewusst rationales beziehungsweise regelgeleitetes Handeln modellieren, stellen zwar zunächst einen geeigneten Ausgangspunkt für die Annäherung an die Beantwortung der Frage dar, wann eine Äußerung als Bewertung aufzufassen ist. Da diese Modelle allerdings Handlungen als isolierte Einzelphänomene betrachten und von regelgeleiteten oder stets bewusst rational handelnden Individuen ausgehen, sowie dazu tendieren, die Oberfläche, Materialität und Medialität sprachlicher Handlungen auszublenden, reichen sie nicht aus, um die Vielschichtigkeit, Dynamik, Situations- und Kontextbezogenheit sowie den interaktionalen und kontingenten Charakter von Bewertungsprozessen in Gesprächen, wie sie dieser Arbeit zugrunde liegen, adäquat zu beschreiben. Aus diesem Grund werden in Abgrenzung und Ergänzung zu den genannten handlungstheoretischen Ansätzen in Verbindung mit dem Konzept der kommunikativen Praktiken auch interaktionslinguistische Arbeiten skizziert, die auf konversationsanalytischen Untersuchungen von Gesprächen in alläglichen und institutionellen Zusammenhängen basieren und vor allem den sequenziellen Verlauf von Bewertungsinteraktionen in den Fokus nehmen.

\subsection{Bewerten als sprachliches Handeln}

Was es bedeutet, etwas sprachlich zu bewerten, und welche Schritte im Zuge des Bewertungsprozesses von den Beteiligten durchlaufen werden, fasst Ripfel (1987) in einem Bewertungsmodell zusammen, mithilfe dessen sie Bewerten anhand von zwei von ihr selbst konzipierten schriftlichen fiktiven Dialogen illustriert und wie folgt definiert:

2 Darunter finden sich zum Beispiel schriftliche Dialoge (Adamzik 1984), Leserbriefe (Herbig/ Sandig 1994), Zeitungsartikel (Sandig 1994) oder Leitartikel in deutschen und finnischen Zeitungen (Tiittula 1994). 
Eine Person (BS) bewertet $\mathrm{zu}$ einem bestimmten Zeitpunkt $\mathrm{t}_{1}$ einen Bewertungsgegenstand (BG), indem BS BG im Hinblick auf bestimmte durch die Vergleichsbasis (V) vorgegebene Bewertungsaspekte (BA) anhand diesen zugeordneten Einordnungsskalen (ES) einordnet und die Einordnungsergebnisse (EE) relativ zu in V vorgegebenen Sollergebnissen (SE) verbunden mit einer Gewichtung (G) auszeichnet. Dabei gilt:

BS ist das bewertende Subjekt.

BG ist der Gegenstand, der vom bewertenden Subjekt zu einer bestimmten Zeit $\mathrm{t}_{1}$ bewertet wird; dabei ist $t_{1}$ für den Handlungsablauf selbst uninteressant; $t_{1}$ spielt nur insofern eine Rolle, als das Bewertungssubjekt denselben Bewertungsgegenstand zu einer anderen Zeit $t_{n}$ anders bewerten kann, weil sich die Wertvorstellungen und damit die Vergleichsbasis des bewertenden Subjekts ändern können.

$\mathrm{V}$ beinhaltet die Wertvorstellungen, an denen der Bewertungsgegenstand gemessen wird. Diese sind beim Bewertenden vorgängig vorhanden; sie werden z. T. unbewußt im Rahmen der Sozialisation, z. T. bewußt durch Lernen erworben. Erst wenn das bewertende Subjekt mit dem Bewertungsgegenstand konfrontiert wird, wirken sie als Vergleichsbasis. ${ }^{3}$

(Ripfel 1987: 155)

In Ripfels Bewertungsmodell sind viele Parallelen zu den Modellierungen von Bayer erkennbar, der einer Bewertung die folgenden Aspekte zuschreibt (Bayer 1982: 16-17): Ein zentrales Merkmal von Bewertungen ist, dass es einen Bewertungsgegenstand geben muss, der bewertet wird. Des Weiteren müssen Alternativen vorliegen, die im Rahmen der Bewertung zum Vergleich herangezogen werden können, und der Vergleich „erfolgt immer gemäß einer bestimmten Hinsicht (unter einem bestimmten Aspekt)“ (Bayer 1982: 16, Herv. im Original). Der Vergleich eines Bewertungsgegenstands mit seinen Alternativen in einer bestimmten Hinsicht führt zu einer in ihrem Komplexitätsgrad variierenden „Ordnung von Gegenstand und Alternativen“ und letztendlich zu einem Sollwert (Bayer 1982: 16, Herv. im Original). Wertung wird erreicht, indem der Sollwert als eine zu erstrebende Größe innerhalb einer Ordnung letztere „zu einer Rangordnung macht“ (Bayer 1982: 16, Herv. im Original). Bewerten heißt also Bayer (1982: 17) zufolge, den Sollwert eines Gegenstands mit seinem Istwert (hinsichtlich eines bestimmten Aspekts) zu vergleichen.

Hartung (2000: 121) beschreibt, ähnlich wie Ripfel (1987) und Bayer (1982), Bewerten als einen Vorgang, bei dem einem Objekt von einem Bewertungssubjekt auf einer sich zwischen den Polen ,negativ` und ,positiv erstreckenden Skala ein bestimmter Wert zugeordnet wird. Die Wertzuweisung geht aus einem Abgleich des Bezugsobjekts der Bewertung mit einem Sollwert hervor (vgl. Hartung 2000:

3 Zur Vergleichsbasis merkt Ripfel an, dass diese entweder institutionell, sozial, staatlich oder subjektiv vorgegeben sein kann (vgl. Ripfel 1987: 155). 
121). Auch Tiittula (1994: 227) stuft Bewerten im Allgemeinen als eine Handlung ein, bei der das Bezugsobjekt die Zuschreibung eines negativen oder positiven Wertes erfährt. Diese Wertzuschreibung ist an der sprachlichen Oberfläche erkennbar, während die von Ripfel und Bayer skizzierten, der bewertenden Person zugeschriebenen mentalen Prozesse von den Beteiligten nicht notwendigerweise expliziert werden müssen.

Sager (1982: 40) beschreibt Bewertungen als Haltung, die ein Bewertender hinsichtlich eines Bezugsobjekts einnimmt, und die ebenfalls auf einer Skala zwischen den Polen ,negativ‘ und ,positiv‘ angesiedelt ist. Die Haltung des Bewertenden gegenüber dem bewerteten Objekt bezeichnet Sager (1982: 40) als Präferenzdisposition, die dem Bewertenden als Basis für die Einstufung - das heißt die bewusste und spezifische Zuschreibung eines Wertes - des betreffenden Objekts sowie der Einordnung ,in eine bestimmte Bewertungsdimension“ dient. Eine Bewertungshandlung wird dann vom Bewertenden vollzogen, wenn dieser seine Wertzuschreibung verbalisiert (vgl. Sager 1982: 40). Auch Keller (2008: 3) differenziert im Rahmen des Bewertens zwischen dem mentalen Prozess, ein evaluatives Urteil zu fällen, und der Verbalisierung des Ergebnisses dieses Prozesses - der Bewertung - in Form einer „evaluativen Aussage“.

Aus den oben angeführten Begriffseingrenzungen lässt sich nun vorerst schlussfolgern, dass Bewerten ein (mentaler) Prozess ist, in dessen Vollzug ein (konkretes oder abstraktes) Objekt von einem Subjekt einer Einstufung auf einer Wertskala, die sich zwischen den Polen negativ und positiv erstreckt, unterzogen wird, und der in einer verbalisierten Bewertung als Ergebnis der vom Subjekt vorgenommenen Einstufung mündet. Bei diesem Prozess spielen verschiedene Einflussgrößen eine Rolle, darunter

(1) Bewertungsaspekte und deren Eigenschaften, hinsichtlich derer ein Objekt auf der Bewertungsskala eingeordnet wird,

(2) der Sollwert, also die Erwartung des Subjekts hinsichtlich des Bewertungsobjekts,

(3) die Vergleichsbasis, aufgrund derer das Subjekt zu einer bestimmten Bewertung eines Objekts kommt, und

(4) der Istwert, der das Ergebnis der Bewertung eines Objekts durch ein Subjekt darstellt.

Die oben dargestellten Modellierungen, die primär auf schriftlichen Kommunikaten oder fiktiven Dialogen basieren, sind für eben diese Bereiche nachvollziehbar, meines Erachtens aber nicht eins zu eins auf mündliche Kommunikation, wie sie in dieser Arbeit untersucht wird, übertragbar. Denn inwiefern die Beteiligten jeden der zuvor genannten Schritte bewusst reflektieren, lässt sich, sofern dies von ihnen nicht konkret verbalisiert wird, nur vermuten, wenn sich eine 
Analyse von nicht-schriftlichen Bewertungen beziehungsweise Bewertungsinteraktionen daran orientiert, was an der sprachlichen Oberfläche der konkreten Äußerungen der Beteiligten beobachtbar ist, Abstand von daraus abgeleiteten mentalen Zuschreibungen und Hintergründen nimmt und die Materialität sowie Medialität der Sprache nicht aus dem Blick verliert (vgl. auch Feilke/Linke 2009).

\subsubsection{Explizite und implizite Bewertungen}

Bewertungen können von den Beteiligten explizit unter Zuhilfenahme sprachlich evaluativer Elemente, beispielsweise explizit evaluativer Phrasen (Sager 1982: 56) oder mittels Bewertungsausdrücken wie Adjektiven, Substantiven und Verben (Sandig 1979: 141-143) realisiert werden. Wenn das im Zuge des Bewertens erreichte Einstufungsergebnis verbalisiert wird, ist nach Tiittula (1994: 228, in Anlehnung an Sandig 1991) davon auszugehen, dass eine explizite Bewertung vorliegt. Implizite Bewertungen können sprachliche Formen aufweisen, die unter anderem Beschreibungen, Feststellungen oder Fragen gleichen (vgl. Adamzik 1984: 241). Sie enthalten nicht notwendigerweise explizit evaluative (lexikalische) Elemente, sind aber mitunter in ihrer Proposition sprachlich so gestaltet, dass „die spezifische Formulierung der Proposition die Funktion übernimmt, die sonst der explizit valuativen Phrase zugekommen wäre“ (Sager 1982: 56).

Keller (2008: 14) spricht nicht von impliziten oder expliziten Bewertungen, sondern führt aus, dass Bewertende zwei Möglichkeiten haben, eine Bewertung zu äußern, nämlich zum einen durch den „semantischen Modus“, das heißt die Wahl von Wörtern, die explizit evaluierend sind und der Haltung des Bewertenden Ausdruck verleihen, oder zum anderen durch den „pragmatischen Modus“, die Wahl solcher Wörter ohne evaluativen Bedeutungsanteil. Bedingung für die Wahl des pragmatischen Modus ist, dass die Situation beziehungsweise der Kontext der geäußerten Bewertung in einem ausreichenden Maße deutlich macht, dass der Bewertende den Rezipienten der Bewertung „,zu weitgehenden interpretativen Schlüssen bewegen möchte“ (Keller 2008: 14). Auch Adamzik führt an, dass eine Zuordnung einer Äußerung als Bewertung nicht allein auf Basis der zur Realisierung der Bewertung genutzten sprachlichen Mittel getroffen werden kann, sondern dass sowohl der situative und sprachliche Kontext als auch das Norm- und Wertesystem der Beteiligten dafür ausschlaggebend ist, inwiefern eine Äußerung als Vollzug einer Bewertung zu deuten ist und ob es sich um eine positive oder negative Bewertung handelt (vgl. Adamzik 1984: 244). Das bedeutet, dass Wertung nicht nur auf Basis einzelner sprachlicher Elemente festzumachen ist, sondern gerade auch der Einbezug des vorliegenden Kontexts sowie - 
bei mündlich realisierten Bewertungen - der Einbezug para- und nonverbaler Phänomene wichtige Orientierungsressourcen darstellen.

\subsubsection{Bewertungen und Begründungen}

Die in der bisher referierten Forschungsliteratur modellierten mentalen Prozesse (vgl. unter anderem Sandig 1993: 165) im Rahmen des Bewertens werden von den Beteiligten oftmals unhinterfragt zugrunde gelegt. Das heißt, dass der der Bewertung zugrunde liegende Einstufungsprozess als „mentale Operation“ (Hartung 2000: 120) nicht notwendigerweise immer verbal expliziert wird (vgl. auch Baldauf-Quilliatre 2012: 211 sowie Fienemann 2006: 36). Neben Baldauf-Quilliatre (2012) verweist auch Hartung (2000: 120) darauf, dass das explizite Verbalisieren einer Wertzuweisung durch einen Wertausdruck nur eine unter vielen Möglichkeiten darstellt, wie Bewertungen interaktional von den Beteiligten realisiert werden. ${ }^{4}$ Auch Fiehler (1990: 30) weist im Zusammenhang mit der allgemein zentralen Rolle, die er Bewertungen und (bewertenden) Stellungnahmen zuschreibt, darauf hin, dass die Aufgaben der Bewertung und Stellungnahme, die „von den Beteiligten - auf verschiedenen Ebenen der Allgemeinheit und in bezug [sic!] auf sehr unterschiedliche Gegebenheiten - individuell oder interaktiv zu lösen sind“, entweder als „private, mentale Bewertungen für sich behalten werden (obwohl sie häufig das weitere Interaktionsverhalten strukturieren und aus ihm erschließbar sind) oder interaktionsrelevant manifestiert werden“. Im Gegensatz zur individuellen mentalen Lösung der Bewertungsaufgabe, die nicht in der Interaktion expliziert wird, wird die interaktive Lösung von den Beteiligten „im gemeinsamen Herausarbeiten einer (möglicherweise geteilten) Bewertung oder Stellungnahme“ vollzogen (Fiehler 1990: 30).

Wenn Wertmaßstäbe oder der (mentale) Bewertungsprozess, der einer Bewertungsäußerung vorangeht, von den Beteiligten in der Interaktion kaum oder gar nicht explizit angeführt werden, ist die in den in Kapitel 2.1 skizzierten Modellen vorherrschende Annahme, dass die Bewertenden bei jeder von ihnen

4 Auch mit Blick auf das Bewertungsobjekt merkt Hartung (2000: 122) an, dass der Prozess der Zuweisung eines Wertes keineswegs expliziert werden muss. Das zu bewertende Objekt kann zum einen bereits eingeführt worden sein, sodass ein Verweis durch eine Proform genügt, um darauf Bezug zu nehmen (siehe auch Kapitel 7 und 8 in dieser Arbeit), zum anderen können sich das Bewertungsobjekt sowie der Zuweisungsprozess situativ ergeben (Hartung 2000: 122). Fienemann (2006: 38) verweist mit Bezug auf Erzählungen darauf, dass Bewertungsmaßstäbe oftmals nicht expliziert, sondern „bereits im vorangehenden Diskurs durch eine Maxime verbalisiert" werden. 
getroffenen Bewertung bewusst reflektiert abwägen, wie sie zu der von ihnen letztendlich geäußerten Gesamtbewertung hinsichtlich eines Objekts gelangt sind, kritisch zu betrachten. Anstatt den Interagierenden auf Basis teils fiktiver schriftlicher Dialoge stets „bewusste, zweckrationale Intentionalität“ (Deppermann/Feilke/Linke 2016: 16) ihres Handelns zu unterstellen, ist der Annahme Garfinkels folgend meines Erachtens davon auszugehen, dass

die Fähigkeit von jemandem, ,rational` zu handeln - d. h. die Fähigkeit von jemandem, während er seine Alltagsangelegenheiten betreibt, alternative Handlungspläne zu berechnen, zu projizieren, die Bedingungen, unter denen er den einen oder den anderen Plan verfolgt, vor dem tatsächlichen Ereignisverlauf auszuwählen, aus einer Auswahl von Mitteln aufgrund seiner technischen Effektivität einem den Vorzug zu geben und so weiter, von der Tatsache abhängt, dass er dazu fähig sein muss, eine große Bandbreite von Merkmalen der sozialen Ordnung buchstäblich als selbstverständlich anzusehen und in sie zu vertrauen. Um das eine Zehntel seiner Situation, das wie ein Eisberg über dem Wasser liegt, rational zu behandeln, muss er fähig sein, die neun Zehntel, die darunter liegen, als unfraglich und, interessanter noch, als einen unbezweifelbaren Hintergrund von Dingen, die nachweislich relevant für seine Kalkulation sind, aber die erscheinen, ohne bemerkt zu werden, zu behandeln. ${ }^{5}$

(Garfinkel 1960/2012: 56-57, Herv. im Original)

Auf die Tatsache, dass Bewertungsmaßstäbe von den Sprechern nicht immer expliziert werden und Bewertungen in unterschiedlichem Maße begründet werden, wird auch in den in diesem Kapitel angeführten handlungstheoretischen Bewertungsmodellen aufmerksam gemacht. So merkt beispielsweise Bayer (1982: 17) an, dass Bewertungen oftmals nicht vollständig verbalisiert und dass sie in großen Teilen realisiert werden, ohne dass dies den Bewertenden bewusst ist. Das Verbalisieren von Begründungen im Zuge von Bewertungen stellt für ihn ein Explizieren des der Bewertung zugrunde liegenden, von den Bewertenden allerdings nicht immer reflektierten und diesen auch nicht immer bewussten Vergleichs dar (vgl. Bayer 1982: 23). Auch Keller (2008: 11) führt aus, dass die Standards, nach deren Maßgabe eine Person zu einer Bewertung kommt, nicht unbedingt explizit genannt werden müssen.

Die Beobachtung, dass lediglich die explizite Äußerung eines Wertes in Bezug auf ein Objekt im Rahmen einer Bewertung ausreichend sein kann (vgl. Hartung 2000: 121-122), um einen Bewertungsaspekt und die damit zusammenhängenden ausschlaggebenden Eigenschaften und ihre Ausprägungen implizit zu kommunizieren, wirft die Frage auf, inwiefern die Verbalisierung des

5 Vergleiche auch Habscheid (2016: 127) sowie Fiehler (1990: 29-30, Herv. im Original), der davon ausgeht, dass Interagierende oftmals eine äußerst große Anzahl spezifischer Aufgaben vollziehen, ohne dass sie sich dessen bewusst sind und „ohne daß sie die Verfahren angeben könnten, mit denen sie die Aufgaben bearbeiten und lösen“. 
einer geäußerten Wertzuweisung zugrunde liegenden Abwägungsprozesses in Form einer Begründung der Wertzuweisung nötig ist, um der Bewertung (allgemeine) Gültigkeit zu verleihen. Hinsichtlich der Gültigkeit von Bewertungen erscheint mit Ripfel (1987: 172-173) zum einen die Relevanz der Kompetenzen der bewertenden Person ein ausschlaggebender Faktor zu sein, das heißt, inwiefern der bewertenden Person unter anderem aufgrund ihrer „Erfahrungen, Kenntnisse oder Fähigkeiten [. . .] ein Urteil zugetraut wird, das für andere verbindlich ist“ (Ripfel 1987: 172). Ein weiterer relevanter Faktor ist zum anderen die rationale Erklärbarkeit und Begründbarkeit von Bewertungen (vgl. Ripfel 1987: 173).

\begin{abstract}
Werturteile erheben den Anspruch auf Verbindlichkeit, aber nicht in dem Sinne, daß der Bewertende diesen Anspruch als Gesetz versteht, dem man folgen muß, sondern der Anspruch leitet sich aus der Begründbarkeit ab. Die Möglichkeit der Geltung von Werturteilen liegt in ihrer Begründung, und diese kann m. E. nur darin bestehen, die Bewertung zu erläutern, die Vergleichsbasis offenzulegen. Das bedeutet, daß mit Werturteilen ein Anspruch auf Gültigkeit erhoben wird, der aber erst durch ihre Anerkennung von seiten anderer erfüllt wird, und diese Anerkennung kann durch Begründung der Werturteile und damit durch eine kommunikative Auseinandersetzung und Einigung erreicht werden.
\end{abstract}

(Ripfel 1987: 174)

Im Falle von Geschmacksäußerungen, die allein auf persönlichen Vorlieben gründen, erweisen sich eine rationale Begründung und eine darauf beruhende Anerkennung der Bewertung durch andere möglicherweise als schwierig. Zwar können, so Ripfel (1987: 174), auch Geschmacksurteile wie Werturteile begründet werden, die der Geschmacksäußerung zugrunde gelegten Wertvorstellungen müssen jedoch für andere nicht relevant sein. Folglich müssen sich Werturteile und Geschmacksäußerungen, zumindest was ihre sprachliche Form angeht, nicht voneinander unterscheiden, sondern lediglich durch die Reichweite ihres Geltungsanspruchs und die Art und Weise, wie sie begründet werden (Ripfel 1987: 174). ${ }^{6}$ Ähnlich fasst auch Keller (2008) den Unterschied zwischen Geschmacksurteilen ${ }^{7}$ und evaluativen Urteilen im Rahmen von Bewertungen. Unter einem evaluativen Urteil versteht er das aus einer „reflektierenden Abwägung“ hervorgegangene Ergebnis, zu dem ein Sprecher durch das Einnehmen einer billigenden oder missbilligenden Haltung bezüglich eines Gegenstands im weiteren Sinne hinsichtlich spezifischer Merkmale kommt (vgl. Keller 2008: 3, 10). Zudem betont Keller (2008: 11) im Hinblick auf Geschmacksurteile, ${ }^{8}$ dass diese nicht mit

6 Vergleiche auch Marschall (2011: 105-106), der davon ausgeht, dass als objektiv lediglich das gelten kann, „was innerhalb der menschlichen Wahrnehmungsmöglichkeiten und einem daraus resultierenden Konsens unabhängig vom interpretierenden Subjekt als zutreffend gilt“.

7 Vergleiche dazu auch Knape (2016: 159, unter Berufung auf Kant).

8 Keller (2008: 11) bezeichnet Geschmacksurteile auch als „gustatorische Urteile“. 
dem Anspruch verbunden sind, „rational diskutierbar noch auf irgendeine Weise begründbar zu sein“, sondern dass die dem Geschmacksurteil zugrunde liegenden Wahrheitsbedingungen einzig und allein dem Sprecher zugänglich sind. ${ }^{9}$ Da ein Geschmacksurteil ein Ausdruck eines „inneren Erlebnisses“ ist, macht es laut Keller (2008: 11) wenig Sinn, einem solchen Urteil zu widersprechen.

\subsubsection{Bewertungen, Beschreibungen und Tatsachenfeststellungen}

Dass Bewertungen Ausdruck subjektiver Sprechereinstellungen sind und subjektive „Stellungnahmen“ (Bayer 1982: 16) enthalten, haben die bisherigen Ausführungen gezeigt. Die Auffassung Bayers (1982: 16), dass Bewertungen aus eben diesem Grund nicht wie etwa Tatsachenbehauptungen als wahr oder falsch bezeichnet, sondern höchstens für bestimmte Personen oder Personengruppen in variierendem Maße plausibel oder akzeptabel sein können, teilt auch Ripfel. Für Ripfel (1987: 164) sind die Kriterien der empirischen Verifizierbarkeit oder logischen Wahrheit ein Indiz dafür, dass es sich um eine Tatsache handelt, wohingegen „Werte auf menschlichen Satzungen oder Konventionen“ fußen, die einem stetigen Wandel unterliegen und nicht wie Tatsachen behandelt werden können. Hartung (2000: 128) sowie Herbig und Sandig (1994: 63) merken an, dass Wertmaßstäbe immer auch von Aspekten der Persönlichkeit, der Identifikation mit bestimmten Gruppen oder von eigenen Überzeugungen und Ideologien beeinflusst sind und so auch Aufschluss über die bewertende Person geben. ${ }^{10}$

Dass es im Rahmen des Bewertens zu einer Differenz von Wertvorstellungen kommen kann, ist aufgrund der genannten Einflüsse, denen Werte unterliegen, nachvollziehbar. Diese Differenz kann sich mit Ripfel (1987: 171-172) beispielsweise im kommunikativen Austausch äußern, indem ein Gesprächspartner durch Nachfragen den anderen Gesprächspartner zu einer Offenlegung seiner Wertvorstellungen auffordert. Inwieweit eine Offenlegung von Wertvorstellungen tatsächlich eingefordert wird, ist sicherlich stark von situativen und

9 Deskriptive Urteile (Beschreibungen wie zum Beispiel „Der Wein ist rot“) können laut Keller (2008: 3) hingegen durch andere Personen hinsichtlich ihres Wahrheitswertes überprüft werden.

10 Vergleiche dazu auch Adamzik (1984: 254, Herv. im Original), die festhält, dass Bewertungen auch eine Art persönliches Sprecherbekenntnis darstellen, denn im Gegensatz zu Beschreibungen oder Feststellungen, die in erster Linie „etwas über die Wirklichkeit und zumindest für das alltägliche Empfinden - erst mittelbar oder sekundär auch etwas über den Sprecher selbst aussagen“, kommt in Bewertungen die persönliche Position des Sprechers viel stärker zum Ausdruck. 
kontextuellen Faktoren abhängig und unterliegt gegebenenfalls insitutionellen Vorgaben und Zwängen. Insofern ist auch die Feststellung Adamziks (1984: 253) zu hinterfragen, dass aufgrund einer nicht möglichen Herbeiführung von „Übereinstimmung in Werturteilen durch rationale Argumentation“ Dissens vorerst eine „unaufhebbare Andersartigkeit oder Fremdheit“ indiziert, die nicht notwendigerweise von den Beteiligten aufgelöst werden kann oder muss. Dies gilt gleichermaßen für Zilligs (1977: 313) Beobachtung, dass eine mit Bewertungen verbundene mögliche Differenz der Sprechermeinungen beziehungsweise -haltungen und die möglicherweise daraus resultierende Face- und Beziehungsbedrohung zwischen den Beteiligten im Falle des Beharrens eines Gesprächspartners auf einer Meinung zu einer Beendigung des Gesprächs durch den anderen Gesprächspartner führen kann. Ob ein Gespräch aufgrund von nicht überwindbarem Dissens von einem der Gesprächspartner abgebrochen wird, hängt womöglich auch mit dem Vertrautheitsgrad der Beziehung ${ }^{11}$ sowie dem Gesprächsmodus und den Selbst- und Fremdpositionierungen der Beteiligten zusammen. Kommt es dennoch zu einem Gesprächsabbruch, stehen den Beteiligten nach Zillig (1977: 313) die folgenden drei Verfahrensweisen zur Verfügung: Entweder sie beharren auf ihrer Bewertung, ohne diese weiter zu begründen, sie einigen sich auf die Bewertung eines Gesprächspartners oder sie erklären die Bewertung für irrelevant und initiieren einen Themenwechsel.

Eine weitere Frage, die im Zusammenhang mit Bewertungen in der Forschungsliteratur diskutiert wird, betrifft die Differenzierung zwischen Bewertungen und Beschreibungen, die sich bei weitem nicht so einfach darstellt, wie es die Unterscheidung von Bewertungen und Tatsachenfeststellungen durch das Subjektivitäts- und Objektivitätskriterium (siehe Kapitel 2.1.3) suggeriert. Dies hat zur Folge, dass Beschreibungen von den Beteiligten teilweise als Bewertungen aufgefasst werden. Eine Lösung des Problems der Differenzierbarkeit zwischen Bewertungen und Feststellungen bietet Zillig (1977: 311) mit dem Vorschlag, mithilfe des „,ich finde nicht'-Kriteriums“ zu überprüfen, ob es sich um eine Bewertung oder ähnliche sprachliche Handlungen handelt. Zillig (1977: 312) kommt zu dem Schluss, dass das „,ich-finde-nicht'-Kriterium“, also die Möglichkeit, auf eine Äußerung mit „Das finde ich (aber) nicht“ zu antworten, die oben genannte Differenzierung zwischen Bewertungen und Beschreibungen ermöglicht. ${ }^{12}$ Gleichwohl räumt Zillig ein, dass die Anwendung des vorgeschlagenen Kriteriums nicht ganz

11 Für eine ausführliche Betrachtung des Zusammenhangs zwischen Bewertungen und der Beziehung der bewertenden Sprecher zueinander siehe Zillig (1977: 314-316).

12 Zillig (1977: 311) führt die Äußerung „C hat 10 Minuten gesprochen“ als Beispiel an, bei dem die Antwort „Das finde ich überhaupt nicht“ nicht möglich ist. Somit handelt es sich bei der Äußerung für Zillig nicht um eine Bewertung. 
unproblematisch ist, da das von ihm genannte Beispiel „C lispelt doch ziemlich stark“ auch als Beschreibung aufgefasst werden könnte (Zillig 1977: 311-312). Die Ebene der möglichen paraverbalen Realisierungvarianten solcher Äußerung blendet Zillig jedoch aus. Zu letzterem Beispiel merkt Zillig (1977: 312) außerdem an, dass er davon ausgehe, dass es sich dabei um ein Handlungsschema des Deutens handelt, das in einem engen Zusammenhang mit dem Bewerten steht, führt dies aber nicht weiter aus. Hinsichtlich der Differenzierbarkeit von Bewertungen und Beschreibungen mittels des „,finde-ich-nicht“-Kriteriums“ weist Zillig darauf hin, dass Bewertungen, „die über Präsuppositionen Bedingungen für andere Sprechakte bilden [. . .] als implizite Bewertungen“ aufgefasst „und gesondert behandel[t]“ werden können (Zillig 1977: 312, Herv. im Original). Ein solches objektiv anmutendes Verfahren zur Unterscheidung von Bewertungen und Beschreibungen, wie es Zillig vorschlägt, erscheint allerdings vor dem Hintergrund der nachfolgend referierten Forschungsliteratur zu kurz gegriffen. So verweist Adamzik (1984: 40, 247) beispielsweise darauf, dass eine eindeutige Abgrenzbarkeit von Bewertungen, Beschreibungen und Feststellungen nicht möglich ist, da es sich um fließende Übergänge handelt, die durch die Sprecher selbst in Form von Aushandlungen unterschiedlicher Deutungen indiziert werden und die die Sprecher gleichzeitig für sich nutzbar machen können, indem sie durch eine nicht eindeutige Positionierung (Adamzik spricht von „Verschleierung“) ihre kommunikativen Ziele effektiver realisieren können. Tiittula (1994: 229) gibt mit Bezug zum Verhältnis von Bewertungen und Beschreibungen $\mathrm{zu}$ bedenken, dass Subjektivität insofern eine Rolle spielt, als je nach den jeweils vorliegenden „Wissensbeständen, Normen, Wertsystemen und Bewertungsmaßstäben“ verschiedene Rezipienten (von Texten) „sehr unterschiedliche Schlüsse aus ,rein informativen“ Äußerungen ziehen“ und der Explizitheitsgrad von Äußerungen vom jeweiligen Rezipienten abhängt. Die Schwierigkeit der Abgrenzung von Beschreibungen und Bewertungen bestätigt sich auch für den Kontext der Kunstkommunikation: Thim-Mabrey (2007: 104) kommt auf der Basis von Analysen schriftlicher Konzertkritiken zu dem Ergebnis, „dass vielfach in einer gewählten Benennung oder in dem Versuch einer Beschreibung Bewertungen mehr oder weniger gewollt mitschwingen“. Auch Kindt (2007: 64) macht anhand von Musikrezensionen auf das von ihm für Beschreibungsaufgaben als typisch bezeichnete Problem aufmerksam, dass oftmals Beschreibungen und Bewertungen vermischt werden (vgl. auch Kindt 2016).

Anhand der vorgestellten Perspektiven wird deutlich, dass es nahezu unvermeidbar ist, beim Beschreiben eines Objekts ${ }^{13}$ diesem gleichzeitig einen

13 Als Beispiel führt Hartung (2000: 123, Herv. im Original) die „Rede von einem stumpfen Messer, einem ereignislosen Film oder einem monotonen Vortrag“ an. 
Wert zuzuordnen (Hartung 2000: 123; vgl. auch Müller 2012: 129 mit Bezug auf das Sprechen über Kunst). Umgekehrt ist es ebenso denkbar, dass die Verbalisierung einer auf ein Objekt bezogenen Bewertung ,auch als nähere Bestimmung“ der Eigenschaften dieses Objekts gelten kann (Hartung 2000: 123). Dies führt Hartung (2000: 123) zu der Schlussfolgerung, dass eine „grundsätzliche Unterscheidung von Beschreiben und Bewerten“ nicht möglich ist, sodass nur im konkreten Gebrauchskontext bestimmt werden kann, ob es sich bei der jeweiligen Äußerung um eine Beschreibung oder Bewertung handelt. Ob eine Beschreibung von den Beteiligten als Bewertung aufgefasst wird, hängt neben kontextuellen Faktoren auch damit zusammen, ob die Beteiligten über Wissen bezüglich der Struktur beziehungsweise möglicher Realisierungsarten von Bewertungen verfügen und entsprechend kompetent interagieren können (vgl. Hartung 2000: 123).

Die vorangehend dargestellte, nicht eindeutig vorzunehmende Abgrenzung von Bewertungen und Beschreibungen bringt laut Adamzik (1984: 247) den bereits oben angesprochenen Nutzen für die Sprecher mit sich: So können Bewertungen in Form von Beschreibungen realisiert werden und im Gegenzug Beschreibungen auch als Bewertungen umgedeutet werden. Die Realisierung einer Bewertung in Form einer Beschreibung macht den Sprecher weniger angreifbar und dient ihm so möglicherweise auch als Vorsichtsmaßnahme, um mit der mit Bewertungen verbundenen Face-Bedrohung umzugehen. ${ }^{14}$

\subsection{Bewerten in kommunikativen Praktiken}

In Kapitel 2.1 wurde herausgestellt, dass die dort diskutierten handlungstheoretischen Bewertungsmodelle dazu tendieren, Handlungen als isolierte Einzelphänomene situations- und kontextentbunden $\mathrm{zu}$ betrachten und von regelgeleiteten oder bewusst rational handelnden Individuen ausgehen, ohne den inkrementellen Vollzugscharakter von Interaktion in den Blick zu nehmen. Von einer individualistisch-rationalistisch geprägten handlungstheoretischen Sichtweise, wie sie in Kapitel 2.1 dargestellt wurde, Abstand nehmend orientieren sich bestimmte praxeologisch ausgerichtete Ansätze (unter anderem Schatzki 2002) an der konkreten Praxis der Individuen, in der die jeweiligen Handlungen vollzogen werden.

14 Ein ähnliches Verfahren beobachtet Günthner (2000) bei Vorwurfsaktivitäten. Die Realisierung eines Vorwurfs im Frageformat hat für die Beteiligten den Vorteil, sich weniger angreifbar zu machen, da diese Realisierungsform ihnen die Möglichkeit bietet, sich bei Bedarf im Anschluss an eine Vorwurfsäußerung darauf zu berufen, lediglich eine Frage gestellt zu haben (Günthner 2000: 112). 
Während in den in Kapitel 2.1 der vorliegenden Arbeit präsentierten linguistischen rationalistisch-handlungstheoretischen Modellen zumindest methodologisch die Annahme im Fokus steht, dass Handeln sich als subjektiv sinnhaftes, bewusst rationales Verhalten manifestiert, wird in soziologisch-praxistheoretischer Perspektive das implizite Wissen und Können als Voraussetzung für das sinnhafte Verhalten der miteinander in der Praxis Agierenden ernst genommen (vgl. SchulzSchaeffer 2010: 319). Im Gegensatz zu den in Kapitel 2.1 dargestellten Handlungstheorien, die isolierte Einzelphänomene und teils fiktives (schrift-)sprachliches Material zum Untersuchungsgegenstand machen, versucht die Praxistheorie, die nach Schatzki (1997: 284 sowie Schatzki 2016: 30) ${ }^{15}$ eine auf unterschiedlichen Praxiskonzepten fußende Familie von Zugängen umfasst, „mit den Mitteln soziologischer Theorie und Forschung das zu untersuchen, was praktisch geschieht“ (Hillebrandt 2014: 8), das heißt „die Praxis allen anderen Größen vorzuordnen“ (Schüttpelz/Meyer 2017: 159). Aus dieser praxeologischen Perspektive sind - zumindest auf Basis der die verschiedenen Zugänge einenden Kernprinzipien (vgl. dazu ausführlicher Schatzki 1996, 1997, 2016 sowie Schäfer 2016) - folgende Annahmen rationalistisch-handlungstheoretischer Modelle $\mathrm{zu}$ problematisieren (vgl. Habscheid 2016: 132): Zuerst stellt sich hinsichtlich handlungstheoretischrationalistischer Modelle, „die individuelle (Zweck-)Rationalität bzw. konventionell geteiltes (Regel-)Wissen [. . .] als hinreichende Voraussetzung menschlicher Kommunikation begreifen“ die Frage, inwiefern Begrifflichkeiten wie ,Regel“ oder ,Regelfolgen“ bei in Praxis eingebettetem Handeln überhaupt (für die Beteiligten als Regel-Repräsentationen beziehungsweise für die Analysierenden als Regel-Modelle) ausreichend sind (vgl. Habscheid 2016: 127, 132). Auch gerät bei rationalistisch-handlungstheoretischen Analysen mit der Betrachtung isolierter Einzelphänomene tendenziell aus dem Blick, dass das in Praxis eingebundene Handeln „in viel umfassendere soziokulturelle Praktiken eingebettet“ ist, die wiederum einen Hintergrund für die Realisierung des praktischen Handelns darstellen (Habscheid 2016: 133-134). Zudem werden Praktiken und damit verbundene Situationsdeutungen nicht notwendigerweise fortwährend nach einem bestimmten Muster reproduziert, sondern können den Handelnden beispielsweise dazu dienen, „Situationen performativ zu gestalten“, etwa durch situationsbezogene Anpassungen oder indem im Rahmen bestimmter Muster agiert wird, um „auf die Definition einer sozialen Situation strategisch Einfluss zu nehmen“ (Habscheid 2016: 134). Praktiken zeichnen sich entsprechend zugleich durch Regelmäßigkeit und Unregelmäßigkeit aus

$15 \mathrm{Zu}$ einer ausführlicheren Darstellung siehe unter anderem Hillebrandt (2014), Schatzki (1996, 1997, 2016), Schäfer (2016) und Schulz-Schaeffer (2010). 
(Habscheid 2016: 134, mit Bezug zu Hörning/Reuter 2004: 13). Ein weiterer Kritikpunkt, der rationalistisch-handlungstheoretischen Modellen (wie unter anderem den in Kapitel 2.1 skizzierten) aus praxeologischer Perspektive entgegengebracht wird, betrifft „die Art und den Grad von Rationalität im Alltag“ (Habscheid 2016: 135). Entgegen der Annahmen rationalistisch-handlungtheoretischer Modelle machen rationale Kalküle, Reflexion und Kontrolle im Alltag nur einen kleinen Teil der Interpretation und Handlungssteuerung aus, der nur möglich ist, weil deren größter Teil auf unhinterfragten Routinen und Annahmen beruht. Aus der Perspektive der Praxistheorie verfügen die Handelnden über bestimmte Kompetenzen, die ihre Teilnahme an Praktiken möglich machen (vgl. Habscheid 2016: 136) und die zum Beispiel als „verkörpertes Wissen“ (Schulz-Schaeffer 2010: 323) bezeichnet werden, das sich im situationsgebundenen Vollzug des Handelns der Akteure zeigen kann, das die Akteure selbst aber außerhalb des Vollzugs ihres Handelns nicht ,als begrifflich formulierbares Wissen oder als Beschreibung der Verfahrensweisen ihres Könnens besitzen“ (Schulz-Schaeffer 2010: 324; vgl. auch Deppermann/Feilke/Linke 2016: 7). Praktiken sind somit „Prozesse verkörperten Betragens [.. .], deren jeweiliger Sinn ganz oder zu (großen) Teilen auf einem durch Handeln präsupponierten Hintergrund von für selbstverständlich gehaltenen Gegebenheiten beruht“ (Habscheid 2016: 137). Ein weiteres Problem rationalistisch-handlungstheoretischer Modelle betrifft schließlich das Ausblenden des Stellenwerts der Interaktion und damit der Tatsache, dass die Beteiligten bei ihrem alltäglichen, in Praxis eingebundenen Handeln bei der Realisierung ihrer Handlungsziele im Alltag zumeist auf das Mitwirken anderer Individuen angewiesen sind (vgl. Habscheid 2016: 136 sowie Schüttpelz/Meyer 2017). Wie können nun diese, in den skizzierten rationalistisch-handlungstheoretischen Beschreibungsmodellen auftretenden Probleme aus praxeologischer Perspektive gelöst werden?

Um der kontingenten, situations- und kontextgebundenen Praxis, in der die Beteiligten handeln, gerecht zu werden, schlagen Schüttpelz und Meyer (2017) ein praxeologisches Glossar vor, das nachfolgend kurz skizziert werden soll. Mit diesem Glossar versuchen sie, sich der „Grobheit [des] Vokabulars für praktische, und d.h. für kooperative und improvisierte Abläufe“ anzunehmen (Schüttpelz/Meyer 2017: 157). Das Glossar umfasst die Begriffe ,Kooperation“ ,Interaktion‘, ,Praktiken', ,Handlung‘, ,Routine‘, ,Technik‘ und ,Praxis‘, die in ihrem Zusammenhang wie folgt dargestellt werden: Kooperation definieren Schüttpelz und Meyer (2017: 158) als die wechselseitige Verfertigung gemeinsamer Ziele, Mittel oder Abläufe, die wiederum Interaktion als wechselseitige Verfertigung gemeinsamer Abläufe entstehen lässt, deren Wiederholbarkeit als Routine definiert wird (Schüttpelz/Meyer 2017: 158). Wechselseitig verfertigte gemeinsame Abläufe bezeichnen Schüttpelz und Meyer (2017: 158) als Praktiken. Das in 
Praxis (als das in einer wechselseitigen Verfertigung befindliche Geschehen) eingebettete Handeln ist wiederum die von den Beteiligten wechselseitig realisierte Verfertigung gemeinsamer oder nicht-gemeinsamer Ziele (vgl. Schüttpelz/ Meyer 2017: 158). Technik ist die wiederholbare Verfertigung der Wiederholbarkeit (wechselseitig verfertigter) gemeinsamer Abläufe (Schüttpelz/Meyer 2017: 158). Diese Definitionen bringen die folgenden theoretischen Implikationen mit sich (Schüttpelz/Meyer 2017: 158-159): Kooperation wird durch Praxis hervorgebracht und Interaktion durch Kooperation. Die Hervorbringung von Praktiken geschieht durch Interaktion, die Hervorbringung von Handlungen durch Praktiken. Die Voraussetzung für Handlungen sind demnach Interaktionen, die ihrerseits wiederum eine Voraussetzung für die Hervorbringung von Praktiken sind (vgl. Schüttpelz/Meyer 2017: 163). Das Hervorbringen von Routinen erfolgt durch Handlungen sowie Praktiken, und Techniken werden schließlich sowohl durch Routinen als auch durch Handlungen und Praktiken hervorgebracht (vgl. Schüttpelz/Meyer 2017: 159). Sie entstehen, ,indem und wenn die wechselseitig verfertigten gemeinsamen Abläufe (i.e. Praktiken) durch ihre Wiederholbarkeit (i.e. Routinen) zu wechselseitig verfertigten gemeinsamen Zielen (i. e. Handlungen) gemacht werden“ (Schüttpelz/Meyer 2017: 162). Schließlich ist bezogen auf das von Schüttpelz und Meyer vorgeschlagene Glossar noch festzuhalten, dass die vorangehend erwähnten Mittel, Ziele und Abläufe „fortlaufend und wechselseitig das eine und das andere für einander [sind]“, sodass eine Trennung der Kategorien weder sinnvoll noch möglich ist (Schüttpelz/Meyer 2017: 157).

Einen ähnlichen praxeologischen Beschreibungsansatz zur Rekonstruktion von Handeln-in-Praxis, in dem wie bei Schüttpelz und Meyer (2017) Kooperation nicht notwendigerweise gemeinsame Ziele beziehungsweise Konsens voraussetzt, schlägt Goodwin (2018) mit seinem Konzept der Co-Operative Action vor. Das Besondere an Goodwins, auf all seinen bisherigen Vorarbeiten fußendem Konzept der Co-Operative Action ist seine Annahme, dass menschliche ${ }^{16}$ Interaktion insofern ko-operativ („,co-operative“) ist, als jede Handlung der Interagierenden Material aus der vorangegangenen Interaktion umfasst, das für die eigene Handlung nutzbar gemacht beziehungsweise transformiert wird (vgl. auch Bergmann 2018: 238).

Co-operative action provides an alternative, quite general mechanism, for both accumulation and incremental change, one lodged within the interstices of mundane action itself. Not only does subsequent action include within its own organization materials created by

16 Vergleiche zu einer Kontrastierung von Goodwins Konzept der menschlichen Co-Operative Action und dem Konzept der Kooperation im biologischen Verständnis mit Bezug zu Tieren ausführlicher Goodwin (2018). 
predecessors, but it also transforms those materials in the ways required for adaptation to current circumstances. This is made possible by the ways in which participants not only attend to, but actively participate in, the detailed organization of each other's action as it unfolds through time.

(Goodwin 2018: 7)

Das Material aus der vorangegangenen Interaktion, das die Beteiligten für ihre eigenen Handlungen nutzen, muss nicht notwendigerweise aus der aktuellen, in Ko-Präsenz verfertigen Interaktion stammen. So vertritt Goodwin (2018: 245) die These, dass dieses Material auch von Vorgängern (,earlier actors“/,predecessors“) stammen kann, die nicht (mehr länger) ko-präsent sind: „However, within the framework of current analysis, what is crucial is how those in the here-and-now are performing operations on materials inherited from someone else, whether dead or alive“" (Goodwin 2018: 250).

Wie bereits vorangehend angedeutet, geht es Goodwin $(2018: 5,6)$ bei seinem Konzept der Co-Operative Action nicht um Kooperation in einem biologisch-anthropologischen Sinne, bei dem der Nutzen beziehungsweise das Wohl der Beteiligten (auf eigene Kosten) sowie das Erzielen von Konsens in den Fokus rücken, sondern Handeln kann in Goodwins Verständnis auch kooperativ sein, wenn die Beteiligten nicht daran arbeiten, Übereinstimmung zu erzielen beziehungsweise wenn nicht-gemeinsame (Handlungs-)Ziele im Vordergrund stehen (vgl. dazu auch Schüttpelz/Meyer 2017). Co-Operative Action beschreibt den Prozess „of building something new through decomposition and reuse with transformation of resources placed in a public environment by an earlier actor“ (Goodwin 2018: 3). In Goodwins Verständnis muss dies also weder notwendigerweise Übereinstimmung implizieren, noch muss es sich um joint action handeln (vgl. Goodwin 2018: 6). Das heißt, dass zum Beispiel auch bei einer konfrontativen beziehungsweise von Nichtübereinstimmung geprägten Interaktion nach Goodwin von Co-Operative Action gesprochen werden kann, da die Beteiligten in ihren Äußerungen auf wechselseitig bereitgestellte, unter Umständen auch auf geteilte oder gemeinsame Ressourcen zurückgreifen, dabei allerdings eindeutig unterschiedliche (gegenläufige) Handlungen realisieren (vgl. Goodwin 2018: 6). Insofern bietet sich Goodwins Ansatz vor allem auch als Hintergrundfolie für die Rekonstruktion der interaktiven Verfertigung nicht übereinstimmender Bewertungen in den dieser Arbeit zugrunde liegenden Gesprächen an.

Goodwin (2018: 149) zeigt unter anderem anhand von Bewertungsinteraktionen in privaten Alltagsgesprächen auf, wie die Beteiligten lexikalische Elemente (wie Adjektive oder Intensivierer, siehe dazu auch Kapitel 7) als projizierende Ressourcen nutzen, um ihren Erfahrungsabgleich und ihre Position hinsichtlich eines Evaluandums dynamisch zu verändern. Anders als die in Kapitel 2.1 dargestellten rationalistisch-handlungstheoretischen Modelle 
hebt Goodwin (2018: 144, 149-150) bei seinen Analysen den Stellenwert der Prosodie im Rahmen von Bewertungen als „embodied assessment“ und Ressource hervor, die es auch nicht direkt am Erleben des bewerteten Ereignisses beziehungsweise der bewerteten Erfahrung Beteiligten erlaubt, an der „embodied appreciation“ des bewerteten Ereignisses zu partizipieren.

Eine Orientierung an Goodwins Konzept der Co-Operative Action eignet sich für die Rekonstruktion von Spezifika der Bewertungsinteraktionen in dieser Arbeit, da Bewertungen über ihre Betrachtung als isolierte Einzelphänomene (siehe Kapitel 2.1) hinaus als „unfolding process“ (Goodwin 2018: 151) nachgezeichnet und Bewertungsinteraktionen als interaktives Organisieren von (Rezeptions-)Erfahrungen durch ko-operatives Handeln (vgl. Goodwin 2018: 149) entfaltet werden können. Bei einem solchen Vorgehen ist es zugleich möglich, die den Interaktionspartnern zugänglichen „public practices“ (Goodwin 2018: 7), mit denen sie ihre Handlungen vollziehen und sich gegenseitig ihr Verständnis der Interaktion anzeigen, in die sie gerade involviert sind, am konkreten sprachlichen Material nachzuzeichnen. Somit wird eine differenziertere Beschreibung der Dynamik des Vollzugscharakters beziehungsweise des von den Beteiligten in wechselseitiger Verfertigung (vgl. Schüttpelz/Meyer 2017) hervorgebrachten Bewertens, der damit verbundenen musterhaften Ausprägungen sowie der von den Interagierenden genutzten, von ihnen selbst allerdings in großen Teilen unreflektierten (vgl. unter anderem Hanks 1996: 231) Methoden ermöglicht. Der „Prozesscharakter der interaktiven Bedeutungskonstitution in sozialen Aktivitäten“ (Günthner 2011: 122) wird dabei - im Vergleich zu den in Kapitel 2.1 diskutierten handlungstheoretischen Modellierungen - im Auge behalten. Ausgehend von Gesprächsdaten als „der primäre materiale Bezugspunkt“ (Deppermann 2000: 104) für die Rekonstruktion interaktiver Bedeutungskonstitutionen der Beteiligten eignet sich - um den kulturellen Wissenshintergründen der an den Gesprächen Beteiligten sowie den Spezifika des untersuchten Gesprächstyps Rechnung zu tragen zudem das von Deppermann (2000) vorgeschlagene Konzept einer ethnografisch angereicherten Gesprächsanalyse, das bei der Interpretation von Gesprächsdaten das von den Beteiligten im Gespräch vorausgesetzte und relevant gemachte, aber nicht explizit dargestellte Wissen (siehe Kapitel 2.3.3) als wichtige Interpretationsressource, um das Handeln der Beteiligten adäquat rekonstruieren zu können, berücksichtigt.

Anknüpfend an die in diesem Kapitel vorgestellten praxeologischen Konzepte und mit Blick auf die in dieser Arbeit untersuchten Gesprächsdaten werden nach Deppermann, Feilke und Linke (2016) Praktiken zunächst in einem mikrostrukturell-gesprächsanalytischen Zugang als Teile von Handlungen und als notwendiger Bestandteil, um letztere zu realisieren (vgl. Deppermann/Feilke/Linke 
2016: 13$)^{17}$ verstanden und untersucht, ohne dabei die soziokulturellen Zusammenhänge, die mit den Gesprächen verbunden sind, zu vernachlässigen. Vor dem Hintergrund der Kulturspezifik der untersuchten Gespräche (siehe Kapitel 1) spielt in Teilen zudem, wie an einigen Gesprächsausschnitten in Kapitel 4.2 und 8 deutlich wird, der makrostrukturell-gattungstheoretische Kontext eine Rolle, in dem Praktiken unter Berücksichtigung der mikrostrukturell-gesprächsanalytischen Ebene als „semiotische, interaktive Großformen des Sprachgebrauchs“ (Deppermann/Feilke/Linke 2016: 12), wie etwa kommunikative Gattungen, in den Blick genommen werden. Kommunikative Gattungen sind nach Luckmann (1989: 38) die Beteiligten orientierende Gesamtmuster, die einen Teil des gesellschaftlichen Wissensvorrats und des kommunikativen Haushalts einer Gesellschaft ausmachen. Wie auch kommunikative Praktiken werden kommunikative Gattungen interaktiv hervorgebracht (vgl. Ayaß 2011: 280) und sind in der konkreten kommunikativen Handlungssituation durch typische Abläufe erkennbar (Luckmann 1989: 38). Somit bieten sie den Beteiligten Lösungen für spezifische kommunikative Probleme (Luckmann 1989: 38), sodass diese nicht jedes Mal neue Wege beschreiten müssen, sondern auf bereits verfestigte und bewährte Muster zurückgreifen können (vgl. Ayaß 2011: 278). Die Entlastung, die kommunikative Gattungen den Beteiligten durch ihre Musterhaftigkeit bieten, entbindet sie davon, Routineprobleme stets neu aushandeln zu müssen, und ermöglicht ihnen somit die Zuwendung zu „neuen Problemen“ (Günthner/Knoblauch 1994: 700; vgl. auch Luckmann 2006). ${ }^{18}$ Die aus makrostrukturell-gattungstheoretischer Perspektive auf Praktiken in den Blick genommenen Großformen hängen zum einen mit bestimmten gesellschaftlich verankerten Konventionen, wie etwa Höflichkeitskonventionen, und mit sozialen Strukturen, zum Beispiel Rollen oder Milieus, zusammen, zum anderen sind sie „nur in der sozial bestimmten performativen Qualität des Vollzugs durch unterschiedliche Akteure mit gattungsspezifischen Beteiligungsrollen fassbar“ (Deppermann/Feilke/Linke 2016: 12).

Praktiken, die aus einer superstrukturell-handlungsfeldbezogenen Perspektive in einem weiten Sinn als potenziell ganze Handlungsfelder umfassend verstanden werden (vgl. Deppermann/Feilke/Linke 2016: 12), stehen bei der mit dieser Arbeit vorgelegten Untersuchung nicht im Fokus, ebenso wenig wie eine historische Betrachtung zeitlicher Veränderungen des Untersuchungsgegenstands dieser Arbeit.

Nachdem im Vorangehenden bereits mehrfach betont wurde, dass das Bewerten als sich interaktiv entfaltender Prozess von den Beteiligten hervorgebracht

17 Siehe Deppermann, Feilke und Linke (2016) zu einer Darstellung der mit der Rezeption des Praktiken-Begriffs in der Linguistik verbundenen Differenzierung von Zugängen.

18 Siehe zu einer ausführlichen Darstellung spezifischer Merkmale von kommunikativen Gattungen unter anderem Luckmann (1989) sowie Günthner und Knoblauch (1994). 
wird, soll nun im Folgenden auf gesprächsanalytische Forschungsarbeiten geblickt werden, die sich bereits sehr ausführlich mit Bewertungsinteraktionen in privaten und institutionellen Settings auseinandergesetzt haben.

\subsection{Bewerten in der Interaktion}

Sequenzielle Strukturen von Bewertungsinteraktionen in privaten und institutionellen Zusammenhängen und die von den Beteiligten zur Realisierung der Bewertungsinteraktionen genutzten Praktiken wurden in zahlreichen konversationsanalytischen Arbeiten detailliert beschrieben und bieten deshalb einen wichtigen Orientierungspunkt für die in der vorliegenden Arbeit vorgenommene Analyse von Bewertungsinteraktionen in einem spezifischen situativen Umfeld und spezifischen Kontexten.

In den nachfolgenden zwei Kapiteln werden zentrale konversationsanalytische Forschungsarbeiten zu Bewertungsinteraktionen in privaten Alltagsgesprächen und institutionellen Zusammenhängen in ihren Kernpunkten skizziert. Die beiden Bereiche Alltagskommunikation und institutionelle Kommunikation sind für diese Arbeit deshalb interessant, weil die in Kapitel 8 analysierten Gespräche beide Bereiche zumindest in Teilen berühren: Die Gespräche finden innerhalb einer privaten Gruppe von miteinander vertrauten Personen statt, gleichzeitig aber an einem der Öffentlichkeit prinzipiell zugänglichen Ort, was bedeutet, das Gesprächsinhalte potenziell von anderen Theaterbesuchern gehört werden können beziehungsweise Gesprächsinhalte auf ein potenziell zuhörendes Publikum zugeschnitten werden. ${ }^{19}$ Da die Gespräche in der inneren Ausgestaltung ihres Ablaufs wenig restringiert sind, dennoch einigen durch die Institution des Theaters vorgegebenen äußeren Bedingungen unterliegen (zum Beispiel der zeitlichen Begrenzung der Theaterpause, siehe dazu Kapitel 4 sowie Kapitel 8), werden sie in dieser Arbeit als semi-institutionell verstanden. Bevor zu Beginn von Kapitel 2.3.2 als einführender Überblick ausführlicher Spezifika institutioneller Kommunikation skizziert werden und im Anschluss einige konversationsanalytische Studien zum Bewerten in institutionellen Zusammenhängen vorgestellt werden, soll im folgenden Kapitel zuerst der Blick auf einige grundlegende Arbeiten zu Bewertungsinteraktionen in privaten Alltagsgesprächen gerichtet werden.

19 Vergleiche Drew und Heritage (1992: 27) zum Sprechen für ein „lauschendes“ Publikum in institutionellen Settings. 


\subsubsection{Bewertungsinteraktionen in Alltagsgesprächen}

Für eine Betrachtung der Realisierung und Aushandlung von Bewertungen in der alltäglichen mündlichen Face-to-face-Interaktion zwischen Freunden und Familienmitgliedern bieten die Untersuchungen von Pomerantz (1975, 1984) sowie von Goodwin und Goodwin (1987, 1992), basierend auf Daten aus dem amerikanischen Englisch, und die Untersuchung von Auer und Uhmann (1982), die sich auf deutsche Gespräche stützen, einen geeigneten Ausgangspunkt. Während sich Pomerantz sowie Auer und Uhmann bei ihren Analysen auf Audioaufnahmen fokussieren, beziehen Goodwin und Goodwin (1987: 4-5) neben Audio- auch Videoaufnahmen und damit multimodale Daten in ihre Analyse ein, die bei einem Familienessen, einem Garten-Picknick und einem Telefongespräch aufgezeichnet wurden. Mit einer Dokumentation von Interaktionen einer Gruppe afroamerikanischer Kinder beim Spielen auf der Straße (vgl. Goodwin/Goodwin 1987: 153) rücken im Gegensatz zu den oben genannten Untersuchungen von Pomerantz sowie Auer und Uhmann neben Gesprächen zwischen Erwachsenen auch solche zwischen Beteiligten jüngerer Altersgruppen in den Fokus. Den Datenbeispielen bei Pomerantz (1984) ist zu entnehmen, dass diese ebenfalls aus alltäglichen Interaktionssituationen (,unspecified context“, Kotthoff 1993: 194) stammen, allerdings wird nicht explizit auf die Datengrundlage verwiesen.

Pomerantz (1984) stellt im Rahmen ihrer Untersuchung von Bewertungsinteraktionen in alltäglichen (privaten) Kommunikationssituationen fest, dass eine Erstbewertung eine Reaktion des Interaktionspartners relevant setzt, die in vielen Fällen in Form einer Zweitbewertung erfolgt (vgl. auch Auer/Uhmann 1982: 3). Dabei ist davon auszugehen, dass - zumindest teilweise - bereits durch die Form der geäußerten Erstbewertung eine Präferen $z^{20}$ für die Art der Zweitbewertung indiziert wird, das heißt, dass der Sprecher einer Erstbewertung den Rezipienten entweder zu einer übereinstimmenden oder nicht übereinstimmenden Zweitbewertung „einlädt““ (Pomerantz 1984: 64). Für Pomerantz (1984: 65) manifestiert

20 Vergleiche zum Präferenzbegriff in der Konversationsanalyse auch Stukenbrock (2013: 234, Herv. im Original): „In der Konversationsanalyse bezeichnet der Terminus Präferenz ein gesprächsorganisatorisches Ordnungsprinzip, wonach Paarsequenzen so organisiert sind, dass bestimmte zweite Paarteile systematisch gegenüber alternativen zweiten Paarteilen begünstigt werden. Der strukturell bevorzugte, präferierte zweite Paarteil stellt die unmarkierte Variante dar und schließt sich prompt an den ersten Paarteil an (Einladung - Annahme; Bitte - Erfüllung). Der dispräferierte zweite Paarteil stellt die markierte Variante dar (Einladung - Ablehnung; Bitte Zurückweisung): Er wird mit mehr Aufwand realisiert und durch Verzögerungssignale, Pausen, Vorankündigungen, Erklärungen usw. gerahmt. Elemente, die einen dispräferierten zweiten Paarteil ankündigen, nennt man Dispräferenzmarker.“ 
sich die Struktur übereinstimmender und nicht übereinstimmender Zweitbewertungen folgendermaßen:

1. Agreements have agreement components occupying the entire agreement turns; disagreements are often prefaced. 2. Agreements are accomplished with stated agreement components; disagreements may be accomplished with a variety of forms, ranging from unstated to stated disagreements. Frequently disagreements, when stated, are formed as partial agreements/partial disagreements; they are weak forms of disagreement. 3. In general, agreements are performed with a minimization of gap between the prior turn's completion and the agreement turn's initiation; disagreement components are frequently delayed within a turn or over a series of turns. 4. Absences of forthcoming agreements or disagreements by recipients with gaps, requests for clarification, and the like are interpretable as instances of unstated, or as-yet-unstated, disagreements.

Die Analyse ihres Datenkorpus führt Pomerantz (1984: 65-70) hinsichtlich der Übereinstimmung mit einer Erstbewertung zur Differenzierung von drei Arten gleichlaufender Zweitbewertungen, die sie als upgrade, same evaluation und downgrade bezeichnet. Upgrades können sich gegenüber dem evaluierenden Ausdruck in der Erstbewertung entweder durch stärker evaluierende Elemente oder durch einen den evaluativen Ausdruck modifizierenden Intensivierer auszeichnen (Pomerantz 1984: 65). Sequenziell betrachtet können Zweitbewertungen in Form von upgrades als starke Zustimmung erachtet werden (Pomerantz 1984: 66). Sie erscheinen oft als Teile von Bündeln oder Serien der Übereinstimmung (Pomerantz 1984: 66). Von einer same evaluation als Zweitbewertung ist laut Pomerantz (1984: 66-67) dann die Rede, wenn sich der Rezipient dem Erstbewertenden anschließt, indem er den vorangehenden evaluativen Ausdruck beispielsweise wiederholt, als „second in a like series“ kennzeichnet oder auf Proformen zurückgreift, die die geäußerte Erstbewertung aufgreifen und Entsprechendes indizieren. Same evaluations können nach Pomerantz (1984: 67) im Gegensatz zu upgrades sowohl bei Übereinstimmung als auch als Komponenten bei Nichtübereinstimmung auftreten. Da einige same evaluations in Pomerantz' Daten von den Sprechern regelmäßig als Vorlaufelement bei Nichtübereinstimmung gewählt werden, können sie in diesem Zusammenhang auch als eine Art schwache Übereinstimmung gelten (1984: 68). Neben upgrades und same evaluations sind downgrades die dritte mögliche Realisierungsart von Zweitbewertungen. Downgrades stellen abgestufte Zustimmung dar und zeichnen sich - im Vergleich zur vorangehenden Bewertung - durch abgeschwächte evaluative Elemente aus, treten zumeist in Begleitung von Verzögerungselementen auf und ziehen möglicherweise eine Modifikation der Erstbewertung nach sich (vgl. Pomerantz 1984: 68, 70; vgl. auch Auer/Uhmann 1982: 4-5, 10-15). Auer und Uhmann (1982: 21-24) zufolge können Modifikationen von Erstbewertungen sowohl durch das Ändern einer Bewertung, zum 
Beispiel in Form einer Relativierung, als auch durch das Ändern des Bezugsobjekts der Bewertung von den Sprechern vorgenommen werden. Wird das Bezugsobjekt der Bewertung verändert, so werden lediglich Teilaspekte des ursprünglichen Bezugsobjekts in den Blick genommen, was eine Veränderung des Geltungsbereichs der monierten Bewertung zur Folge hat (Auer/Uhmann 1982: 22). Das Hervorbringen von Argumenten zur Untermauerung der Erstbewertung und um die Gesprächspartner doch noch zur Zustimmung zu bewegen, stellt eine weitere Möglichkeit der Modifikation dar (vgl. Auer/Uhmann 1982: 22). Nach Pomerantz (1984: 68) rufen abgestufte Zustimmungen häufig Sequenzen der Nichtübereinstimmung hervor. Als mögliche Reaktion auf eine solche abgestufte Zweitbewertung können Interagierende ihre Erstbewertung durch eine noch stärkere Bewertung bekräftigen (1984: 68). Da einige downgrades Nichtübereinstimmung hervorrufen können, bezeichnet Pomerantz (1984: 69) diese - neben same evaluations - ebenfalls als eine Art schwache Übereinstimmung. Pomerantz hält auf Basis ihres Datenkorpus Unterschiede in der Art der Realisierung von Nichtübereinstimmungen im Vergleich zu Übereinstimmungen fest. Zum einen kann Nichtübereinstimmung entweder explizit in direktem Kontrast zur vorhergehenden Äußerung verbalisiert werden und beinhaltet dann „exclusively disagreement components“ oder sie wird in Form von schwacher Nichtübereinstimmung ${ }^{21}$ realisiert, die der vorangegangenen Äußerung beispielsweise durch Einschränkungen, Ausnahmen, Ergänzungen usw. teilweise zustimmt und teilweise widerspricht (Pomerantz 1984: 74). Das Einbinden von Verzögerungssignalen stellt laut Pomerantz (1984: 70) dann ein typisches Merkmal nicht übereinstimmender Zweitbewertungen dar, wenn die Form der Erstbewertung zu einer übereinstimmenden Zweitbewertung einlädt. Im Falle einer nicht übereinstimmenden Zweitbewertung greifen Zweitbewertende auf die folgenden zwei Verfahren zurück, obwohl durch die Form der Erstbewertung vom Sprecher eine Präferenz für Übereinstimmung indiziert wird: Sie verwenden zum Beispiel Verzögerungssignale wie Schweigen, fordern mittels Fragen im Vorfeld der Verbalisierung von Nichtübereinstimmung Spezifizierungen ein und/oder inkludieren Komponenten schwacher Übereinstimmung, das heißt partieller Übereinstimmung oder partieller Nichtübereinstimmung (Pomerantz 1984: 75).

Auch Auer und Uhmann (1982) widmen sich, anknüpfend an die Analysen von Pomerantz (1975), auf Basis eines Korpus deutschsprachiger Daten der Untersuchung von Bewertungsinteraktionen in Alltagsgesprächen. Sie unterscheiden

21 Diese Form bezeichne ich im Rahmen meiner Analyse in der vorliegenden Arbeit in Anlehnung an Gruber (1996: 185) als „partiellen Widerspruch“. 
mit Blick auf gleichlaufende Zweitbewertungen in Anlehnung an die von Pomerantz getroffene dreigliedrige Differenzierung zwei Gruppen: Die Intensität einer ersten Bewertung steigernde „Eskalierungen (scaling up)“ und die Intensität einer ersten Bewertung verringernde „Deskalierungen (scaling down)“ (Auer/Uhmann 1982: 4). Eskalierte Bewertungen bezeichnen Auer und Uhmann (1982: 4) durch ihre potenziell sequenzterminierende Kraft als „optimale Reaktionen“ auf Erstbewertungen, Deskalierungen können hingegen als Nichtübereinstimmung oder Projektion von Nichtübereinstimmung möglicherweise dazu führen, dass die Beteiligten eine für sie annehmbarere „Einschätzung“ des Bezugsobjekts aushandeln. Folglich können sie die Expansion einer Bewertungssequenz nach sich ziehen (vgl. Auer/Uhmann 1982: 4).

Zwischen den beiden vorangehend genannten Gruppen der Eskalierungen und Deskalierungen siedeln Auer und Uhman (1982: 4, 8) die „semantisch niveaugleichen“ Zweitbewertungen an, die teils Eskalierungen und teils Deskalierungen gleichen, die Intensität einer Erstbewertung jedoch kaum modifizieren. Abweichend von Pomerantz' (1984) dreigliedriger Gruppierung gleichlaufender Zweitbewertungen beschränken sich Auer und Uhmann (1982: 4-5) allerdings auf die Differenzierung zwischen Eskalierung und Deskalierung. ${ }^{22}$ Diese von Pomerantz' drei Kategorien abweichende Zweiteilung begründen Auer und Uhmann (1982: 29) folgendermaßen: Zum einen liegen Pomerantz' Kategorien uneinheitliche Kriterien zugrunde, da sie im Falle von upgrades und downgrades auf Basis der semantischen Intensität der Bewertungsausdrücke arbeitet. Im Falle von sames spielen inhaltliche Aspekte hingegen keine Rolle. Zum anderen kritisieren Auer und Uhmann (1982: 29), dass bei Pomerantz sames ausschließlich dadurch bestimmt sind, dass „ihr sequenzielles Verhalten entweder dem der Eskalierung oder dem der Deskalierung entspricht“, das heißt sie entweder zu einer Sequenzexpansion oder -terminierung führen. Dies hat eine Unterordnung sequenzieller Gesichtspunkte zugunsten der semantischen Intensität als entscheidender Maßstab zur Folge (vgl. Auer/Uhmann 1982: 29). Auer und Uhmann (1982: 29) plädieren dafür, dass eine konversationsanalytische Untersuchung von Bewertungsinteraktionen in erster Linie „sequenziell beschriebene Kategorien entwickeln und verwenden sollte“. Aus diesem Grund unterscheiden Auer und Uhmann (1982: 29) unter Einbezug der semantischen Intensität und der „sequenzterminierende[n] Kraft“ lediglich die beiden Gruppen der Eskalierung und Deskalierung, den „semantisch dazwischenliegenden niveau-

22 Zu einer ausführlicheren Darlegung der Unterschiede zwischen Pomerantz' Untersuchung und der Analyse von Auer und Uhmann vergleiche Auer/Uhmann (1982: 29). 
gleichen Bewertungen“ weisen sie jedoch - im Gegensatz zu Pomerantz - keine eigene Gruppe zu.

Auer und Uhmann (1982: 29) stellen - wie auch Pomerantz (1984: 63-64) auf Basis ihres Korpus fest, dass übereinstimmende Zweitbewertungen als Reaktion auf eine Erstbewertung in großen Teilen von den Sprechern als präferiert behandelt werden. Aus diesen Ergebnissen leiten Auer und Uhmann (1982: 5) ab, dass das Vermeiden von Nichtübereinstimmung und die Präferenz für Übereinstimmung „ein durchgängiges konversationelles Prinzip“ darstellt. Dies muss, wie Kotthoff (1993: 196) ausführt, allerdings nicht immer der Fall sein, denn „[. . .] preference structures are preshaped by institutional requirements, which are not necessarily shared by everybody, and in turn help to create the institutional setting“. Man denke hier beispielsweise an Confrontainment-Sendungen im Fernsehen, die bei einer vorliegenden Präferenz für Übereinstimmung nicht funktionieren würden.

Während der Produzent einer Erstbewertung mit der Äußerungsgestaltung dieser Bewertung indizieren kann, welche Form der Rezipientenreaktion von ihm präferiert wird, stehen ihm bei der Realisierung einer ersten Bewertung vergleichsweise wenig Ressourcen zur Verfügung, um einschätzen zu können, ob sich sein Gegenüber der Erstbewertung anschließen wird oder nicht (vgl. Auer/ Uhmann 1982: 5). Es besteht also die Gefahr, dass die Erstbewertung „nicht mit einer gleichlaufenden Zweiten Bewertung quittiert wird“ (Auer/Uhmann 1982: 5). Ist dies der Fall, so kann diese potenzielle Meinungsdivergenz zu einer Expansion der Bewertungssequenz führen (vgl. Auer/Uhmann 1982: 5). Die Gefahr einer nicht gleichlaufenden Zweitbewertung spricht für das Argument von Auer und Uhmann (1982: 20), dass Interagierende direkte Nichtübereinstimmung nach Möglichkeit vermeiden. Liegen jedoch divergierende Meinungen vor, so wird diese Nichtübereinstimmung von den Sprechern so versteckt realisiert, dass sie diese stets revidieren können (vgl. Auer/Uhmann 1982: 20). Erkennen beide Interaktionspartner, dass eine Meinungsdivergenz vorliegt, führt dies vielfach zur bereits angesprochenen Expansion der Bewertungssequenz, die der Aushandlung von Übereinstimmung dient (vgl. Auer/Uhmann 1982: 20). Im Rahmen dieser Expansion können die Beteiligten Erstbewertungen, die Nichtübereinstimmung nach sich ziehen, entweder in variierendem Maße modifizieren, sodass diesen doch noch zugestimmt werden kann, oder sie können das Bewertungsobjekt minimal verändern, indem sie „nachträglich nur Teilaspekte“ des ursprünglichen Bezugsobjekts in den Fokus rücken und damit eine Veränderung des ursprünglichen Geltungsbereichs ihrer Bewertung herbeiführen (vgl. Auer/Uhmann 1982: 22-23). Eine weitere Möglichkeit zum Erreichen von Übereinstimmung ist laut Auer und Uhmann (1982: 22) das Anführen von Argumenten, um die Erstbewertung zu stützen. 
Die vorangehenden Ausführungen verdeutlichen, dass Bewertungen keine starren, unwiderruflich festgelegten Konstrukte sind, sondern Bewerten ein dynamischer Prozess ist, in dessen Rahmen Bewertungen ko-produziert und gemeinsam ausgehandelt werden, und der sich situations- und kontextspezifisch entfaltet. Goodwin und Goodwin verweisen auf eben diese Dynamik, indem sie feststellen, dass Bewertungen eine wiedererkennbare, drei-schrittige Struktur aufweisen, die erstens aus einem „peak of involvement“ besteht, dem zweitens „visible precursors of that peak“ vorausgehen, welche von den Gesprächsteilnehmern genutzt werden können, um das Erreichen des „peak of involvement“ zu koordinieren, sowie die drittens Verfahren umfasst, die es ermöglichen, sich vom Zustand erhöhter gemeinsamer Beteiligung zurückzuziehen (1992: 181182). Diese Struktur beeinflusst laut Goodwin und Goodwin (1992: 182, Herv. im Original) maßgeblich, wie am Gespräch Beteiligte mit Äußerungen umgehen:

In order to co-participate in an appropriate fashion at an appropriate moment, recipients track in fine detail the unfolding structure of the speaker's utterance, paying close attention to not only the projective possibilities made available by its emerging syntactic structure (e. g. the type of unit that is about to occur), but also the precise way in which it is spoken (e.g. lengthening of sounds within words and intonation changes). The detailed organization of the talk occurring within a turn thus constitutes a most important aspect of the context that participants are attending to, both to make sense out of what is happening at the moment, and as a resource for the organization of their subsequent action.

Die Orientierung an der sich im Vollzug der Interaktion entfaltenden Struktur von Sprecheräußerungen ermöglicht den Sprechern beispielsweise bei Bewertungen als „projected social actions“ (Lindström/Mondada 2009a: 300), ihre Zweitbewertung bereits vor der Finalisierung der noch laufenden Erstbewertung beziehungsweise parallel zu dieser realisieren zu können (vgl. Goodwin/Goodwin 1992: 162-164). ${ }^{23}$ Bewertungen stellen also, so lässt sich mit Goodwin und Goodwin (1992: 182) schlussfolgern, eine Möglichkeit dar, „where participants negotiate and display to each other a congruent view of the events that they encounter in their phenomenal world“.

Obwohl die vorangehend genannten frühen Arbeiten von Pomerantz, Auer und Uhman sowie Goodwin und Goodwin differenzierte Untersuchungen von

23 Als Beispiel führen Goodwin und Goodwin (1992: 163) den folgenden Gesprächsausschnitt an:

Dianne: Jeff made en asparagus pie

it wz $s$ : : so: goo:d

$\rightarrow$ Clacia: I love it.

$\mathrm{Zu}$ „strategischen Vorteilen“ einer zur Erstbewertung in Überlappung geäußerten Gegenbewertung siehe Auer und Uhmann (1982: 7). Zu anticipatory completion vergleiche Lerner (1996). 
Bewertungsinteraktionen vorlegen und zeigen, wie das Bewerten von den Beteiligten gemeinsam interaktiv realisiert wird, werden in den Analysen vorwiegend Bewertungen vergangener und nicht in situ stattfindender Ereignisse betrachtet (vgl. auch Weidner 2017). Auch weist der Gesprächstyp (Alltagsgespräche) andere Rahmenbedingungen und Spezifika auf (unter anderem hinsichtlich des sequenziellen Ablaufs, zeitlicher Beschränkungen, Rollenkonstellationen, dem Vertrautheitsgrad der Beteiligten usw.), als dies beispielsweise in institutionellen Gesprächen der Fall ist. Das führt dazu, dass teilweise Prinzipien, die für private Alltagsgespräche herausgestellt wurden, ein Allgemeingültgkeitsanspruch unterstellt wird, der so vor allem im Bereich institutioneller Kommunikation nicht gegeben ist (vgl. Brünner 1987: 27-28). ${ }^{24}$

\subsubsection{Bewertungsinteraktionen in institutionellen Gesprächen}

Anknüpfend an die im Vorangehenden aufgezeigte Schwierigkeit, dass sich die in konversationsanalytischen Untersuchungen auf Basis privater Alltagsgespräche herausgestellten Prinzipien (siehe Kapitel 2.3.1) nicht ohne Weiteres auf Gespräche übertragen lassen, die in bestimmten institutionellen Kontexten stattfinden, erfolgt zunächst eine knappe Skizzierung einiger Spezifika institutioneller Kommunikation, um - wie bereits in Kapitel 2.3 angeschnitten - mögliche Parallelen hinsichtlich der Rahmenbedingungen zwischen den in dieser Arbeit untersuchten Theaterpausengesprächen und institutionellen Gesprächen herausstellen zu können. Anschließend werden in diesem Kapitel einige jüngere konversationsanalytische Arbeiten sowie deren Erkenntnisse zum Bewerten in institutionellen Kontexten vorgestellt.

Ehlich und Rehbein verstehen Institutionen als Teilbereiche der Gesellschaft, die entsprechend bestimmten gesellschaftlichen Zwecken dienen (vgl. Ehlich/Rehbein 1977: 36). ${ }^{25}$ Die Institutionalität von Interaktion in diesen Teilbereichen der Gesellschaft ergibt sich laut Drew und Heritage (1992: 3-4; vgl. auch Groß/Harren 2016: 13) allerdings nicht (allein) durch ihr Setting, sondern die Interaktion ist vielmehr insofern institutionell, als die institutionellen oder professionellen Identitäten der Agenten von ihnen für ihr berufliches Handeln relevant gemacht werden (vgl. auch Schegloff et al. 2002: 10-12). Institutionelle Kommunikation ist in der

24 Vergleiche beispielsweise auch Schwitalla (2006: 244) zur institutionellen Spezifik von Nachgesprächen.

$25 \mathrm{Zu}$ einer Darstellung von in der Linguistik untersuchten Institutionen siehe zum Beispiel Brünner (1987). 
Regel geprägt durch ,institutions- und aufgabenspezifisch[e] normativ[e] Erwartungen“ auf Seiten der Klienten und Agenten (Groß/Harren 2016: 9), eine relativ restringierte Zielorientierung sowie spezifische Einschränkungen dessen, was die Beteiligten zur Interaktion beitragen (dürfen) (vgl. Drew/Heritage 1992: 22). Auch können in der Interaktion entwickelte Schlussfolgerungen und Interpretationen von institutionellen Rahmenbedingungen geprägt sein (vgl. Drew/Heritage 1992: 22) sowie Beiträge des Gesprächspartners zudem möglicherweise ,anders behandelt [werden] als in informellen Gesprächstypen“ (Groß/Harren 2016: 14).

Mit der Kommunikation in Institutionen sind unterschiedliche Zugänge zu Wissen sowie unterschiedliche Wissensstatus verbunden (siehe dazu auch Kapitel 2.3.3). Ehlich und Rehbein (1977: 39) nennen in diesem Zusammenhang das Aktantenwissen, das die Handlungen der innerhalb einer Institution Agierenden leitet, und einen Teil der Gesamtheit des Wissens über Institutionen - des Institutionenwissens - ausmacht (vgl. auch Brünner 1987: 24). Unterschiedliche Zugänge zu Wissen und differierende Wissensstatus auf Seiten von Agenten und Klienten als den innerhalb einer Institution interagierenden Personen können Wissensasymmetrien begünstigen (vgl. Drew 2012: 153 sowie Drew/Heritage 1992: 47-53). Diese, auf unterschiedlichen Wissensbeständen gründenden Asymmetrien zu bearbeiten, kann einen kommunikativen Mehraufwand erfordern und möglicherweise Verständigungsprobleme nach sich ziehen (vgl. Groß/Harren 2016: 12). Die Institutionsspezifik sprachlichen Handelns in institutioneller Kommunikation kann sich nach Brünner (1987: 25-26) unter anderem folgendermaßen zeigen: Institutionelle Regelungen können die „Abfolge von Sprechern und Themen“, die „Kontextualisierung sprachlicher Handlungen“ sowie „Sequenzmuster von sprachlichen Handlungen und die Ablaufkonstitution von Interaktionsschemata“ beeinflussen. Auch „Regeln und Verfahren der Verständnissicherung“ können institutionsspezifisch variieren (Brünner 1987: 26).

Die grundlegenden und bereits extensiv erforschten Formen alltäglicher Interaktion stellen als analyseleitender Hintergrund (vgl. Groß/Harren 2016: 16) eine Vergleichgröße dar, auf deren Basis andere Formen formellerer oder institutionellerer Interaktion erkannt und herausgestellt werden können (vgl. Drew/Heritage 1992: 19). Die Untersuchung von Kommunikation in Institutionen kann somit zur Prüfung der Generalisierbarkeit von Ergebnissen, die aus Analysen privater Alltagskommunikation gewonnen wurden (siehe Kapitel 2.2.2), beitragen (vgl. Brünner 1987: 28). Dies zeigen, ausgehend von konversationsanalytischen Arbeiten zu Bewertungsinteraktionen, die im Rahmen privater Alltagsgespräche als Teil von Erzählungen vergangener Ereignisse auftreten (siehe Kapitel 2.2.2), jüngere, auf Videodaten basierende und multimodale Ressourcen berücksichtigende Untersuchungen mit Fokus auf institutionellen Settings, die spezifische Ausprägungen von Bewertungsinteraktionen in beruflichen Kontexten aufzeigen, 
etwa in der häuslichen Altenpflege (Lindström/Heinemann 2009), beim Autokauf (Mondada 2009), bei der Begutachtung von Kleidungsstücken in einem Modeatelier (Fasulo/Monzoni 2009), bei Elternsprechtagsgesprächen (vgl. Wegner 2016) oder in TV-Kochsendungen in einem öffentlich-massenmedialen Kontext (vgl. Weidner 2017). Die Ergebnisse dieser Untersuchungen zeigen, dass Bewertungen beziehungsweise Bewertungssequenzen eingebettet in größere Aktivitäten in Erscheinung treten oder den übergeordneten Zweck einer Aktivität darstellen können, wenn es sich um Aktivitäten handelt, in deren Vollzug die Beteiligten in eine bewertende Praxis involviert sind, oder das Bewerten selbst die zentrale Aktivität bildet (Lindström/Mondada 2009a: 303; vgl. auch Wegner 2016: 15). In letzterem Fall dienen Bewertungen als ein Schlüsselmerkmal zur Realisierung der übergeordneten Aktivität, das von den Beteiligten zur Orientierung genutzt und als solches erwartet wird (Lindström/Mondada 2009a: 303-304). Dies verdeutlicht, dass Bewertungen sowohl den jeweiligen institutionellen Kontext mit konstituieren als auch an diesen angepasst werden (vgl. Lindström/Mondada 2009a: 304). Somit können Bewertungen als Ressource zur Realisierung der Institutionalität des „ongoing talk-in-interaction“ dienen, und stellen auch eine Ressource für die Abschwächung oder Verstärkung dieser Institutionalität dar (Lindström/Mondada 2009a: 304). Für Lindström und Mondada (2009a: 304, in Anlehnung an Sacks 1972) hängen mit der auf den Kontext zugeschnittenen Anpassung von Bewertungen und der Konstitution des Kontexts durch Bewertungen die institutionellen Identitäten der Sprecher und die sozialen epistemischen Positionen zusammen, denn ,[i]n work settings, the rights to assess, as well as the epistemic authority of the assessors, are often related to professional expertise and to institutional membership categories“. Die von Lindström und Mondada genannten Kategorien und Identitäten sowie auch der Kontext werden dynamisch im Zuge der Produktion und Aushandlung von Bewertungen von den Gesprächsbeteiligten aktiv etabliert, beansprucht, zugeschrieben und infrage gestellt, wobei gleichermaßen Übereinstimmung oder Nichtübereinstimmung forciert werden kann (2009a: 304-305). Dies hat zur Folge, dass zum Beispiel nicht von einer Präferenz für Übereinstimmung als unabhängig vom Gesprächskontext geltendes universales Prinzip (vgl. Auer/Uhmann 1982) ausgegangen werden kann, sondern dass vielmehr von den Sprechern selbst mit ihren Äußerungen im jeweiligen Kontext indiziert wird, welche Art der Präferenz vorliegt.

Wenn die in dieser Arbeit untersuchten Pausengespräche auch keiner strikten institutionellen Regelung unterliegen, so sind doch einige, durch die Institution des Theaters gesetzte Rahmenbedingungen zu beachten. Dies betrifft vor allem den Zeitpunkt der Gesprächseröffnung und -beendigung, da die Zuschauergespräche vor und nach der Theateraufführung stattfinden können, die Theaterbesucher jedoch dazu angehalten sind, Gespräche während der Rezep- 
tion des Theaterstücks zu vermeiden (siehe Kapitel 1). Die Gespräche in der Theaterpause sind durch einen festgelegten Zeitrahmen begrenzt, der auf die Gesprächsbeendigung Einfluss nehmen kann (siehe Kapitel 8.4). In diesem Punkt besteht zum Beispiel ein grundlegender Unterschied zu Gesprächen, die vor einem Kunstwerk parallel zu dessen Betrachtung in einem Museum stattfinden und zeitlich nicht beziehungsweise weniger restringiert sind (siehe Kapitel 3.1). Auch der Aspekt der Präsentation und Vermittlung von Wissen im Zusammenhang mit Selbst- und Fremdpositionierungen als Experte oder Laie sowie das Zuschreiben und Absprechen von Wissen spielen im Theaterpausengespräch eine Rolle und unterliegen spezifischen institutionellen Besonderheiten. Damit verbunden können Verfahren der Verständnissicherung (vgl. Brünner 1987: 26), wie zum Beispiel Rückfragen oder selbstinitiierte Belehrungen ohne deren Einforderung, hinsichtlich ihrer Angemessenheit verändert und im Kontext der Kunstkommunikation potenziell Face-bedrohend sein (siehe das folgende Kapitel 2.3.3 sowie Kapitel 7.5).

\subsubsection{Wissensdimensionen und epistemische Autorität}

Mit der Realisierung und Aushandlung einer Bewertung ist zugleich auch die Beanspruchung und Aushandlung einer bestimmten epistemischen Position (vgl. Stivers/Mondada/Steensig 2011: 7-8; vgl. auch Pomerantz 1984: 92) sowie des den Bewertungen zugrunde liegenden Wissens der Beteiligten verbunden. Das Aushandeln von epistemischen Positionen und Wissen ist im prestigeträchtigen Kontext der Kunstkommunikation (siehe Kapitel 1), in dem die in dieser Arbeit untersuchten Bewertungsinteraktionen vollzogen werden, besonders brisant, da mit der Offenlegung von Nicht-Wissen im Zuge des Bewertens durch den Sprecher selbst oder durch seine Gesprächspartner eine potenzielle FaceBedrohung einhergeht (siehe dazu auch Kapitel 2.3.4 und 2.3.5). Das Hintergrundwissen der Interagierenden (common ground) kann von ihnen einerseits durch den Adressatenzuschnitt (recipient design) ihrer Gesprächsbeiträge sowie andererseits durch grounding indiziert werden (Deppermann 2015: 7). Das bedeutet zum einen, dass Sprecher mit der Gestaltung ihrer Äußerungen dem Adressaten auch immer ein bestimmtes Wissen unterstellen (vgl. Deppermann 2015: 7). Von diesen Zuschreibungen beziehungsweise „Annahmen über das Partnerwissen“ hängt ab, wie Äußerungen sprachlich gestaltet werden, um einerseits Verständlichkeit zu gewährleisten, andererseits aber Redundanzen hinsichtlich des Partnerwissens zu vermeiden (Deppermann 2015: 7). Der Terminus ,grounding“ beschreibt einen „Prozess wechselseitiger Verstehensdokumentationen“, der den Interagierenden dazu dient, sich „das für das aktuelle 
joint project relevante und gültige intersubjektive Verständnis“ gegenseitig anzuzeigen und dieses auszuhandeln (Deppermann 2015: 11, Herv. im Original). Der grounding-Prozess läuft im Hintergrund der Interaktion beziehungsweise parallel zu dieser mit (Deppermann 2015: 11).

Um in der Analyse der dieser Arbeit zugrunde liegenden Theaterpausengespräche (Kapitel 8) rekonstruieren zu können, inwieweit die Beteiligten im Rahmen der Realisierung von Bewertungen und Begründungen in den Bewertungsinteraktionen (Nicht-)Wissen relevant setzen und aushandeln, wird im Folgenden der Blick auf die Konzepte epistemischer Status und epistemic stance gerichtet sowie der Zusammenhang zwischen Bewertungen und epistemischen Rechten skizziert.

Beim epistemischen Status handelt es sich nach Deppermann (2015: 14) um „eine soziale Zuschreibung an Interaktionsbeteiligte“. Er ist definiert durch die Rechte, Verantwortungen und Pflichten einer Person, über bestimmtes Wissen zu verfügen (Mondada 2013: 599). Stivers, Mondada und Steensig (2011: 9) unterscheiden im Zusammenhang mit dem epistemischen Status einer Person drei Wissensdimensionen, von denen zumindest zwei auch im Hinblick auf die dieser Arbeit zugrunde liegenden Gespräche eine zentrale Rolle spielen.

Der epistemische Zugang besagt, wer über welches Wissen verfügt, und zwar mit welchem Grad an Gewissheit sowie auf Basis welcher Quellen und welcher Direktheit. Liegt Wissen in Form von type 1 knowables vor, haben die Beteiligten das Recht sowie die Pflicht, über dieses Wissen zu verfügen (zum Beispiel der eigene Name) (vgl. Pomerantz 1980: 187). Liegt Wissen in Form von type 2 knowables vor, so wird von den Beteiligten angenommen, Zugang zu Kenntnissen über Dritte zu haben (zum Beispiel wo sich eine nahestehende Person aufhält, was er/sie am Vortag gemacht hat) (vgl. Pomerantz 1980: 187-188).

Die epistemische Priorität gibt Auskunft über den Wissensvorrang, das heißt, welche jeweiligen Rechte des Wissens und der Inanspruchnahme sowie welche Wissensautorität vorliegen (Stivers/Mondada/Steensig 2011: 9-10; vgl. auch Drew/ Heritage 1992: 49 für institutionelle Kommunikation). Stivers, Mondada und Steensig (2011: 14) verweisen im Hinblick auf die Art des vorliegenden Wissenszugangs auf Labov und Fanshel (1977), die zwischen A event knowledge und B event knowledge unterscheiden, also Wissen, das in erster Linie der Sprecher besitzt (A) oder zu dem primär der Rezipient Zugang hat (B). Mittels der Indizierung, ob es sich um $A$ event knowledge oder $B$ event knowledge handelt, können Sprecher eine abgeschwächte epistemische Position deutlich werden lassen (Stivers/Mondada/ Steensig 2011: 14). Die Art des vorliegenden Wissens steht nach Lerner auch im Zusammenhang mit der Präferenz, für sich selbst zu sprechen, statt andere für sich sprechen zu lassen: 
[. . .] speaking for oneself (as animator and as author/owner of an utterance) seems to be preferred over speaking for another participant (as animator but not as author/owner of an utterance), in the sense that voicing a coparticipant's speaking on his or her own behalf, whereas voicing one's own experiences and the like is not ordinarily an alternative to anything.

(Lerner 1996: 305, Herv. im Original)

$\mathrm{Zu}$ der von Stivers, Mondada und Steensig genannten dritten Dimension der epistemischen Verantwortung gehört unter anderem die Verantwortung zu wissen, welches den Interaktionspartnern gemeinsame Hintergrundwissen vorliegt, die Verantwortung, bestimmte Handlungen (wie beispielsweise das Erfragen von Informationen) nicht $\mathrm{zu}$ vollziehen, wenn die Antworten bereits bekannt sind sowie die Verantwortung, bei der Gestaltung der Äußerung auf ihr Wissen über die Interaktionspartner zurückzugreifen (vgl. Stivers/Mondada/Steensig 2011: 18; vgl. auch Deppermann 2015).

Mit dem epistemischen Status und der Art des Wissenszugangs gehen „epistemische Rechte und Pflichten“ einher, „deren interaktive Relevanz allerdings durch das Verhältnis zwischen den Interaktionsbeteiligten moduliert ist“ (Deppermann 2015: 13). Bezogen auf Bewertungen bedeutet dies, dass die Rechte, die mit einem direkten Zugang zum Bewertungsobjekt verbunden sind, gegenüber Rechten überwiegen, die mit einem nur indirekten Zugang zusammenhängen (Heritage/Raymond 2005: 17; vgl. auch Deppermann 2015: 13 und Pomerantz 1980: 190).

Im Vergleich zum epistemischen Status, der die Art des Wissenszugangs indiziert, geht es beim Einnehmen der epistemic stance um eine Haltung im Hinblick auf einen Wissensgegenstand, die „mit bestimmten sprachlichen Praktiken der Wissenskommunikation zum Ausdruck gebracht wird“ (Deppermann 2015: 14). Mithilfe dieser sprachlichen Praktiken können Interagierende anzeigen, über welches Wissen sie verfügen oder nicht verfügen (vgl. Deppermann 2015: 14). Laut Deppermann (2015: 14) stellt beispielsweise das Anzeigen „des Grades der Gewissheit einer Aussage“ einen zentralen Aspekt der epistemic stance dar. Mondada (2013: 600) zufolge betrifft die epistemische Haltung „the moment-bymoment expression" des Verhältnisses zwischen der Position des Wissens $(\mathrm{K}+)^{26}$ und der Position des Nicht-Wissens (K-) „as managed through the design of turns at talk as well as the format of specific actions“. Das Äußern einer Haltung des Nicht-Wissens lädt den wissenden Gesprächspartner dazu ein, genauere Angaben zu machen und Informationen zu geben, während eine Wissen indizierende Haltung Erzählungen und Belehrungen begünstigt und den Nicht-Wissenden zu einer Bestätigung einlädt (Mondada 2013: 600, siehe auch Kapitel 7.5). Zu berücksichtigen ist in diesem Zusammenhang allerdings, in welchem Kontext

$26 \mathrm{~K}$ steht für knowledge. 
die von Mondada beschriebene Haltung des Wissens oder Nicht-Wissens eingebettet ist, da sowohl eine Offenlegung von Nicht-Wissen als auch eine nicht explizit eingeforderte Demonstration von Wissen, zum Beispiel im Rahmen von Belehrungen, potenziell heikel sein können und von den Beteiligten möglicherweise sanktioniert werden könnten.

Wie insbesondere im Rahmen von Bewertungen epistemische Rechte indiziert und ausgehandelt werden, beschreiben Heritage und Raymond (2005: 19) auf Basis privater Alltagsgespräche für das amerkanische Englisch. Sie stellen heraus, dass deklarative Bewertungen (wie im Deutschen zum Beispiel die Prädikation „X ist $\mathrm{Y}^{\text {“) }}$ einen unmittelbaren Zugang zum Bewertungsobjekt indizieren und keine sprachlichen Merkmale aufweisen, die den mit der Bewertung verbundenen deklarativen Anspruch stärken oder schwächen (vgl. Heritage/Raymond 2005: 19). Ein deskalierter epistemischer Zugang zu einem Bewertungsobjekt beziehungsweise einem Sachverhalt wird mittels einer deskalierten Erstbewertung indiziert (vgl. Heritage/Raymond 2005: 19). Ein an eine Bewertung angeschlossenes Rückversicherungssignal wie beispielsweise ne im Deutschen (vgl. Schwitalla 2002; Heritage und Raymond (2005) sprechen hier von einer tag question) kann dazu dienen, den mit der Erstbewertung verbundenen epistemischen Zugang zu relativieren, indem Erstbewertende einen im Vergleich zum Gesprächspartner vermeintlich nur indirekten Zugang (zweiter Ordnung) zu einem Bewertungsgegenstand indizieren und gleichzeitig ihr Gegenüber zu einer Zustimmung der Bewertung einladen (vgl. Heritage/Raymond 2005: 20). Der Anschluss eines Rückversicherungssignals ist auch bei Zweitbewertungen möglich. Beim Rückgriff auf ein Rückversicherungssignal im Rahmen von Zweitbewertungen ist von einer Umkehr der Funktion im Vergleich zu Erstbewertungen auszugehen: Während in Erstbewertungen eingebettete Rückversicherungssignale das mit der Bewertung beanspruchte epistemische Recht deskalieren, dienen an Zweitbewertungen angeschlossene Rückversicherungssignale dazu, den mit der Zweitbewertung verbundenen epistemischen Zugang zu eskalieren, indem das assessment + tag-Format zu Übereinstimmung der Zweitbewertung einlädt und somit der im Zuge der Erstbewertung indizierten Position zuvorkommt (Heritage/Raymond 2005: 28).

Eskalierte Erstbewertungen werden von Heritage und Raymond (2005: 21) als dritte Ressource zur Indizierung epistemischen Rechts angeführt. Im Rahmen der Realisierung eskalierter Bewertungen schreiben Heritage und Raymond (2005: 21-22) der negativen Interrogativfrage einen besonderen Stellenwert zu: Sie setzt einerseits - mehr als eine Bewertung in Form eines Deklarativsatzes - eine Zweitbewertung konditionell relevant und gibt andererseits die Form der Zweitbewertung durch die Projektion einer Ja-/Nein-Frage bereits vor. Da eine Bewertung in Form einer negativen Interrogativfrage verstärkt zu Übereinstimmung einlädt, indiziert sie eine etablierte oder feststehende Position und damit einhergehend 
auch eine extensivere Vertrautheit mit dem Bewertungsgegenstand und/oder stärkere Rechte, diesen zu bewerten (vgl. Heritage/Raymond 2005: 22). Der Rückgriff auf eine interrogative Syntax ist ebenfalls bei Zweitbewertungen möglich (vgl. Heritage/Raymond 2005: 23). Mit einer Realisierung einer Zweitbewertung in interrogativer Form wird die „Erstheit“ einer Erstbewertung unterminiert und die epistemischen Rechte werden für sich selbst beansprucht (Heritage/Raymond 2005: 23). Die Übertragbarkeit beziehungsweise Gültigkeit der von Heritage und Raymond (2005) auf Basis privater Alltagsinteraktionen gewonnenen Erkenntnisse hinsichtlich der Aushandlung epistemischer Rechte im Zuge des Bewertens bleibt allerdings für andere (zum Beispiel institutionelle oder öffentliche) Kontexte zu klären (siehe Kapitel 2.3.2).

\subsubsection{Stancetaking und soziale Positionierungen}

Auf die zentrale Rolle von mit Bewertungen vorgenommenen sozialen Positionierungen wurde mit Bezug zur Kunstkommunikation bereits in Kapitel 1 verwiesen. Im Folgenden sollen nun Möglichkeiten des stancetaking sowie sozialer Positionierungen - allgemein und speziell im Kontext der Kunstkommunikation systematischer betrachtet werden. Die Auseinandersetzung mit dem Einnehmen persönlicher Haltungen und mit Varianten sozialer Positionierungen ist insofern für die Analyse dieser Arbeit relevant, als davon auszugehen ist, dass die Beteiligten in den Pausengesprächen mit ihren Bewertungen nicht nur Wissen indizieren und zuschreiben (siehe Kapitel 2.3.3), sondern auch bestimmte Selbst- und Fremdpositionierungen vornehmen, aushandeln und gegebenenfalls relativieren.

Nach Lucius-Hoene und Deppermann (2004: 169) beansprucht ein Sprecher mit dem Einnehmen einer bestimmten Haltung, beispielsweise durch eine Bewertung, gleichzeitig eine bestimmte Position in einem bestimmten sozialen Raum und signalisiert den anderen an der Interaktion Beteiligten „wie er gesehen werden möchte“. Als eine typische linguistische Ressource zum Ausdruck von solchen Selbstpositionierungen nennt Kärkkäinen (2006: 700) die Phrase I think, „because it contains reference to the speaker (the first-person pronoun) and a verb that denotes a private or interior cognitive process“ (vgl. auch Finegan 1995). ${ }^{27}$

27 Vergleiche auch die im Rahmen von Bewertungsinteraktionen in den dieser Arbeit zugrunde gelegten Daten oftmals von den Sprechern genutzte Äußerung „ich finde/ich fand“ (Kapitel 8). Kärkkäinen (2006: 700) merkt an, dass „I think“ in der linguistischen Forschung zwar als „prototype of subjective language“ gilt, weist allerdings auch darauf hin, dass „I think“ in ihren Daten aus dem gesprochenen amerikanischen Englisch, die verschiedenen 
Neben dieser Selbstpositionierung kann einem Sprecher aufgrund seiner im Rahmen einer Bewertung kommunizierten Haltung von seinen Interaktionspartnern auch eine Position zugeschrieben werden, die durch die Art des Adressierens des Interaktionspartners und mit „auf ihn bezogenen Handlungen“ indiziert wird (Lucius-Hoene/Deppermann 2004: 169). Positionierungen und stancetaking können mit einem interaktiven Abgleich von Haltungen aller beteiligten Gesprächspartner verbunden sein, der beispielsweise durch interrogative Bestätigungseinladungen (wie im Deutschen zum Beispiel „Findest du nicht auch?“ oder „Ging es dir auch so?“) initiiert werden kann.

Die interaktive Hervorbringung von Haltungen und Positionierungen im Rahmen von Bewertungsinteraktionen beschreibt Kärkkäinen (2006: 718) folgendermaßen:

[S]tance is very often established and negotiated as an interactional practice. The articulation and differentiation of stance is a joint activity between discourse participants, i. e., it essentially involves the recipients' coparticipation. Assessments are one type of action that involve taking up positions and making evaluations, and they indeed often come, not only in pairs, but in longer strings, with each speaker constructing a stance by building on, modifying, and aligning or disaligning with the immediately co-present stance of a dialogic partner.

Welche Rolle das Bewerten für die soziale Positionierung im Kontext der Kunstkommunikation spielt, zeigt unter anderem Hausendorf (2012). Er hält am Beispiel einer Szene aus Woody Allens Film Manhattan aus dem Jahre 1979 fest, dass mit dem Bewerten „eine bestimmte Art der Selbstdarstellung“ verbunden ist, die „als Ausdruck einer bestimmten sozialen Position“ gesehen werden kann (Hausendorf 2012: 95). „Die Art und Weise der Inanspruchnahme dieser Position beim und durch das Reden“ bezeichnet Hausendorf (2012: 95) als „,soziale Positionierung“, die den Interagierenden dazu dient, „sich wechselseitig aufzuzeigen, wer oder was sie sind bzw. als wer oder was sie betrachtet und verstanden werden wollen“. Vor allem die Kunstkommunikation stellt für Hausendorf (2012: 95) einen Ort dar, der es den Kunstrezipienten erlaubt, bestimmte soziale Positionen einzunehmen und diese soziale Positionierung vor allem durch die

Interaktionssettings entstammen, oftmals „in sequential contexts where its referential meaning is quite vague and bleached (to the point of deletion of the first-person pronoun) “ auftritt und deutlich ,as a discourse marker that simply frames an upcoming stance or marks boundaries within a speaker’s speech“ fungiert. Außerdem ist, so Kärkkäinen (2006: 708), „I think“ häufig auch in „second-pair parts of adjacency pairs“, beispielsweise in Antworten, Zweitbewertungen und Meinungsbekundungen oder schwachen Zustimmungen, zu finden, aber auch in „more free-ranging sequential environments“, in denen es um „strategic work“ geht, in dessen Rahmen oftmals auch Face-Belange eine Rolle spielen. 
Äußerung von Bewertungen vorzunehmen (siehe auch Kapitel 1). „Die Ausbildung einer für das Selbstverständnis der modernen Gesellschaft zentralen Kategorie der Selbst- und Fremdwahrnehmung läuft also“, so Hausendorf (2012: 96), „vereinfacht gesagt, auch und gerade über das Bewerten als Teil einer sich entwickelnden kommunikativen Praxis der Kunstkommunikation und den damit sich manifestierenden sozialen Positionierungen“. Allerdings ist nach Kindt (2007: 57) den „Kommunikation über Kunst"-Betreibenden oftmals nicht bewusst, wie extensiv sie und weitere Beteiligte diese Kommunikation nutzen, um sich sozial zu positionieren.

Müller (2012: 129) hält anhand einer Untersuchung, im Rahmen derer in Straßeninterviews Fußgängern ein Selbstprortrait der Künstlerin Frida Kahlo gezeigt wurde, das sie beschreiben und zu dem sie Stellung nehmen sollten (,Was geht der Frau durch den Kopf?“ und „Gefällt Ihnen das Bild?“), fest, dass die Bildbeschreibung durch die Rezipienten „,indirekt darauf ab[zielt], Haltungen zu Kunst im Allgemeinen und soziale Positionierungen ihr gegenüber hervorzubringen, indem nämlich verschiedene Strategien der Beschreibung auch soziale Positionen relativ zum Feld der Kunst markieren“. Dabei zeigen sich soziale Positionen in den von den Rezipienten des Bildes vorgenommenen Beschreibungen „anhand unterschiedlicher Wissenshorizonte und Einstellungen dazu“ (Müller 2012: 131). Bezüglich der verschiedenen Arten des angeführten Wissens unterscheidet Müller (2012: 131) zwischen „kunsthistorischem Spezialwissen“ und „allgemeinem Bildungswissen“ sowie Indikationen von „Laientum“ in Form von expliziten Verbalisierungen von „Kunstferne“. Diese Positionierung als Kunstlaie indizieren die Rezipienten laut Müller (2012: 131-132) unter anderem durch das Bestreiten der „Zuständigkeit, Expertise oder Fähigkeit für die gestellte Aufgabe“ oder durch das Abweichen von der „Domäne der Kunst“ und den Rückgriff auf „alltagsweltliche Beschreibungsstrategien“ sowie die sprachliche Bewältigung „künstlerischer Formensprache“ mittels „Analogiebildung aus der Alltagswelt“ (vgl. auch Johanson/ Glow 2015 im Hinblick auf durch Interviews herausgestellte Verfahren der Bewertung von „non-experts“ im Anschluss an die Rezeption eines Theaterstücks; siehe auch Kapitel 3.2). Expertentum („Habitus des Kunstkenners/der Kunstkennerin“) hingegen wird von den Rezipienten indiziert durch den Rückgriff auf Sachwissen (wie zum Beispiel die Erläuterung des biografischen Hintergrunds der Künstlerin) oder aber durch das Heranziehen „weniger fundierte[n] Sachwissen[s]“ und den „Habitus des gebildeten Laien“ (Müller 2012: 132-134). Ähnlich wie Müller verweist auch Kindt (2007) auf verschiedene Strategien der Positionierung im Rahmen der Kommunikation über Kunst. Hierunter fallen zum Beispiel das Indizieren von Kunst-Nähe oder der Verweis auf Autoritäten, um „das eigene positive Selbstbild stützen“ zu können (2007: 71). 


\subsubsection{Face und Face-Work}

Dass Bewertungen mit einer potenziellen Bedrohung des Face einhergehen können, da eine Rezipientenreaktion auf eine Bewertung nicht immer vorhersehbar ist, wurde vorangehend bereits angesprochen (siehe Kapitel 2.3.1). Auch in der mit Prestige verbundenen Kunstkommunikation (vgl. Müller/Kluwe 2012: 5), in der es um Haltungen, Positionierungen, Selbstdarstellungen und damit auch um Identitäten geht, können unzureichende Begründungen von Bewertungen oder aufgedecktes Nichtwissen beziehungsweise eine dadurch vorgenommene Infragestellung der Kompetenzen des Sprechers von den Gesprächspartnern moniert werden und somit im schlimmsten Fall zu einer Blamage des Sprechers führen. Um im empirischen Teil der vorliegenden Arbeit herausstellen zu können, inwieweit Face für die an der Interaktion Beteiligten eine Rolle spielt beziehungsweise wie sie Face im Gespräch relevant setzen und im Rahmen von Bewertungsinteraktionen und damit verbundenen Positionierungen aushandeln, soll nachfolgend den Fragen nachgegangen werden, was unter dem Konzept ,Face‘ zu verstehen ist und wie in der Interaktion von den Beteiligten Face-Work betrieben werden kann, um mit den oben genannten Gefahren umzugehen.

Die Begriffe Face und Face-Work wurden ursprünglich von Goffman eingeführt, um die rituelle Organisation sozialer Begegnungssituationen zu erklären (Lerner 1996: 303). Goffman (1967: 5) definiert Face als den positiven sozialen Wert, den eine Person für sich selbst beansprucht. Für Brown und Levinson (1987: 61) umfasst Face die zwei Aspekte des positiven Face und des negativen Face. Das positive Face beschreiben sie als das positive, beständige Selbstbild beziehungsweise die Persönlichkeit, die eine Person für sich selbst beansprucht. Mit dem positiven Face ist laut Brown und Levinson (1987: 62) der Wunsch verbunden, dass die eigenen Bedürfnisse auch von zumindest einigen anderen Personen als für erstrebenswert gehalten werden: „The most salient aspect of a person's personality in interaction is what that personality requires of other interactants - in particular, it includes the desire to be ratified, understood, approved of, liked or admired“ (Brown/Levinson 1987: 62). Was als erstrebenswert gilt, ist in hohem Maße kultur- sowie gruppenspezifisch und beruht letztendlich auch auf persönlichen Vorlieben (Brown/Levinson 1987: 63-64). Das negative Face umfasst das Bedürfnis, im Vollzug seiner Handlungen nicht von anderen gestört zu werden (vgl. Brown/Levinson 1987: 62). Mit dem Face einer Person geht auch die (öffentliche) Bewertung dieser einher (Lerner 1996: 303), was dazu führen kann, dass sich eine Person im Kontakt mit anderen Personen - je nachdem ob in der Interaktion die an die Person gerichteten Erwartungen anderer bestätigt werden oder nicht - gut oder schlecht fühlt (Goffman 1967: 6; vgl. auch SpencerOatey 2007: 644). Dies bedeutet zugleich, dass das Face einer Person nicht als 
mit der Person verkörpert zu fassen ist, sondern als etwas, das auf eine diffuse Art und Weise im Fluss der Ereignisse zu verorten ist (Goffman 1967: 7) und sich nur dann manifestiert, wenn die Beteiligten diese Ereignisse einer Bewertung unterziehen (Spencer-Oatey 2007: 642). Welch große Rolle die Gesellschaft bei der Zuschreibung und dem Management von Face für eine Person spielen kann, beschreibt Goffman (1967: 10) folgendermaßen:

In any case, while his social face can be his most personal possession and the center of his security and pleasure, it is only on loan to him from society; it will be withdrawn unless he conducts himself in a way that is worthy of it. Approved attributes and their relation to face make of every man his own jailer; this is a fundamental social constraint even though each man may like his cell.

Diese Feststellung Goffmans lässt darauf schließen, dass Face nicht als statisches Persönlichkeitsmerkmal vorzustellen ist, sondern erst im Kontakt mit anderen (als Vertreter der Gesellschaft) konstituiert und ausgehandelt wird.

Laut Goffman (1967: 10) wird von einem Mitglied einer jeglichen Gruppe sowohl Selbstachtung beziehungsweise die Wahrung des eigenen Selbstbilds sowie die Wahrung des Selbstbildes anderer Personen erwartet. Die Art der gegenseitigen Wahrung beziehungsweise Akzeptanz des Face beschreibt Goffman (1967: 11) als ,a ,working‘ acceptance, not a ,real' one, since it tends to be based not on agreement of candidly expressed heart-felt evaluations, but upon a willingness to give temporary lip service to judgments with which the participants do not really agree“. Durch Handlungen anderer Personen, die den Face-Bedürfnissen des jeweiligen Sprechers oder Adressaten entgegenstehen, kann dessen Face bedroht werden (vgl. Brown/Levinson 1987: 65). Zu den das negative Face einer Person bedrohenden Handlungen zählen Brown und Levinson (1987: 66) Anweisungen und Bitten, Vor- und Ratschläge, Erinnerungen (etwas zu tun), sowie Drohungen, Warnungen, Sanktionen, aber auch Angebote, Versprechen, Komplimente oder starke Emotionsbekundungen. Eine Bedrohung des positiven Face kann hervorgerufen werden durch heftige (negative) Emotionsbekundungen (,,out-of-control' emotions"), durch Respektlosigkeit, das Ansprechen von Tabu-Themen oder durch das Ansprechen von Themen, die in einem gefährlichen Ausmaß zur Emotionalisierung oder Polarisierung verleiten, durch offensichtliches Nicht-Kooperieren, sowie durch eine (unabsichtlich) inadequate Adressierung oder andere Status indizierende Identifikationen von Personen bei ersten Begegnungen (Brown/Levinson 1987: 66-67). Wie gehen nun aber die Beteiligten mit Face-Bedrohungen beziehungsweise Face-Angriffen, wie Brown und Levinson sie beschreiben, um, und auf welche Ressourcen können sie dabei zurückgreifen?

Um einer potenziellen Face-Bedrohung zu begegnen, haben die Interagierenden die Möglichkeit, Face-Work zu betreiben (Goffman 1967: 12). Obwohl die 
Konsequenzen, die die von einer Person ausgeführten Handlungen zum Schutz des Face nach sich ziehen, dieser nicht immer (in vollem Umfang) bewusst sind, werden die Handlungen, so Goffman (1967: 13), oft zu habituellen und standardisierten Praktiken. Jede Person, Subkultur und Gesellschaft scheint über ihr eigenes, charakteristisches Repertoire solcher Praktiken $\mathrm{zu}$ verfügen, das auf ein einziges logisch zusammenhängendes Bezugssystem möglicher Praktiken zurückzugehen scheint: „It is as if face, by its very nature, can be saved only in a certain number of ways, and as if each social grouping must make its selections from this single matrix of possibilities“. Auch SpencerOatey (2007: 649, unter Bezugnahme auf Goffman 1967: 5) weist darauf hin, dass mit dem Management von Face ebenso die Beurteilung bestimmter, kulturspezifisch variierender Werte verbunden ist, so wie auch die von den Angehörigen einer Gesellschaft zugrunde gelegten Konzeptionen sozialer Rechte und Pflichten, die dazu führen können, dass die an einer solchen Interaktionssituation Beteiligten bestimmte Vorkommnisse als Face-Bedrohung auffassen.

Goffman (1967: 15) unterscheidet zwei Strategien, im Rahmen des Face-Work mit einer potenziellen Bedrohung des Face umzugehen: Wenn eine Person den Kontakt zu anderen Personen gänzlich vermeidet, weil Face-Bedrohungen in Kontaktsituationen sehr wahrscheinlich auftreten können, bezeichnet Goffman (1967: 15; vgl. auch Lerner 1996: 309) dies als Vermeidungsstrategie. Sollte es jedoch trotzdem dazu kommen, dass eine Person mit einem solchen, potenziell Facebedrohenden Kontakt konfrontiert wird, so stehen ihr laut Goffman (1967: 16) weitere Möglichkeiten der Vermeidung zur Verfügung, indem sie beispielsweise solche Themen und Handlungen umgeht, ,that would lead to the expression of information that is inconsistent with the line he is maintaining“. Die Person kann also das aktuelle Gesprächsthema oder die Richtung der Handlung wechseln und vorerst jede Gefühlsregung zurückhalten, bis sie sich versichert hat, „,what kind of line the others will be ready to support for him“ (Goffman 1967: 16).

Any claims regarding self may be made with belittling modesty, with strong qualifications, or with a mode of unseriousness; by hedging in these ways he will have prepared a self for himself that will not be discredited by exposure, personal failure, or the unanticipated acts of others. And if he does not hedge his claims about self, he will at least attempt to be realistic about them, knowing that otherwise events may discredit him and make him lose face.

(Goffman 1967: 16)

Aus diesem Zitat Goffmans lässt sich schließen, dass dem Hedging ${ }^{28}$ im Rahmen des Face-Work eine wichtige Rolle zukommt beziehungsweise dass umgekehrt Hedging ein Hinweis auf eine potenzielle Face-Bedrohung sein kann. Die oben

28 Siehe zu einer Definition von Hedging Kapitel 7.3. 
dargestellten, von Goffman als defensiv bezeichneten Maßnahmen zum Schutz des eigenen Face können auch als protektive Strategien zum Tragen kommen, indem eine Person auf bestimmte Ressourcen zurückgreift, um das Face des anderen zu wahren (Goffman 1967: 16): Hierzu zählen ein respektvoller und höflicher Umgang, Diskretion, der Einsatz von Umschreibungen und Verschleierungen und die Formulierung von Antworten mit einer „vorsichtigen Ambiguität“, um so das Face der anderen Person zu wahren, ,even if their welfare is not“".

Neben defensiven und protektiven Strategien zur Wahrung des Face stellt Goffman (1967: 19) mit dem „corrective process“ eine dritte Möglichkeit vor. Im Zuge dieses Prozesses werden Begegnungen, in denen das Face einer oder mehrerer Personen auf dem Spiel steht, als „Bedrohung“, die offizieller Aufmerksamkeit bedarf, behandelt und deren Auswirkungen „korrigiert“ (vgl. Goffman 1967: 19). Die Korrektur umfasst vier Aktivitäten: Eine beteiligte Person wird auf ihren „Fehltritt“ aufmerksam gemacht (vgl. Goffman 1967: 20). Der Person, die den Fehltritt begangen hat, wird die Möglichkeit eingeräumt, diesen durch eine Umdeutung (zum Beispiel einer bedrohlichen Äußerung in ein belangloses Ereignis oder einen Witz) oder den Verweis zu korrigieren, dass die Person unter dem Einfluss (von Personen oder Dingen) stand und nicht Herr ihrer eigenen Äußerung war (vgl. Goffman 1967: 20). Neben diesen beiden Möglichkeiten kann die Person des Weiteren Kompensationsleistungen, „Selbstbestrafung“ oder Wiedergutmachung anbieten (vgl. Goffman 1967: 21). Die Person, deren Face bedroht wurde, kann das Wiedergutmachungsangebot akzeptieren und dem Gegenüber vergeben (vgl. Goffman 1967: 21). In einem letzten Schritt würdigt die Person, die den Fehltritt begangen hat, dass ihr Nachsicht gewährt wurde (vgl. Goffman 1967: 21). Erst wenn dieser Schritt vollzogen ist, gilt der „corrective process“ als beendet (Goffman 1967: 21). Goffman (1967: 21) gibt zu bedenken, dass das geschilderte Vorgehen ein „model for interpersonal ritual behavior“ darstellt, von dem aber in erheblichem Maße abgewichen werden kann. Ebenso verweist er darauf, dass jede zur „Neutralisierung“ einer Face-Bedrohung eingesetzte Praktik die Gefahr mit sich bringt, dass die Bedrohung gewollt eingesetzt wird (Goffman 1967: 24):

When a person treats face-work not as something he need be prepared to perform, but rather as something that others can be counted on to perform or to accept, then an encounter or an undertaking becomes less a scene of mutual considerateness than an arena in which a contest or match is held. The purpose of the game is to preserve everyone's line from an inexcusable contradiction, while scoring as many points as possible against one's adversaries and making as many gains as possible for oneself.

Im Gegensatz zu diesem „aggressiven“ Einsatz von Strategien des Face-Work kann letzteres auch kooperativ betrieben werden (Goffman 1967: 27). Initiator und betreibende Person von Face-Work muss nicht notwendigerweise derjenige 
sein, durch den die Face-Bedrohung ausgelöst wurde, sondern der Mangel an Bemühungen zur Wiedergutmachung seitens einer Person kann durch die Bemühungen anderer kompensiert werden (vgl. Goffman 1967: 27). Kooperative Maßnahmen zur Wahrung des Face können beispielsweise darin bestehen, anderen Komplimente $\mathrm{zu}$ machen, diese für sich selbst aber abzuerkennen (vgl. Goffman 1967: 27).

Lerner (1996: 305, 319) betont, was bereits Goffman indiziert, nämlich dass die Konzepte ,Face‘ und ,Face-Work‘ erst als eine Praktik in der Interaktion konstituiert und ausgehandelt werden, beispielsweise im Zuge des Managements von Präferenz oder bei einer gemeinsamen Vervollständigung von Redezügen (vgl. auch Arundale 2010: 2078-2079). Für Lerner (1996: 319) lässt sich die Organisation von Face-Work nicht etwa durch das Bedürfnis, sein Face zu wahren, erklären, sondern die mit dem Selbst verbundenen Gefühle und ein durch Face ausgedrücktes Selbst werden reflexiv und als Produkte wiedererkennbarer Umstände im Zuge von Aktivitäten im Vollzug der Interaktion erreicht und produziert. Für Spencer-Oatey (2007: 654) ist mit Face eine Vielzahl von Eigenschaften verbunden, die stetig ausbalanciert werden müssen. Zum einen ist Face ein facettenreiches Phänomen, dass gleichzeitig aber auch ein einheitliches Konzept darstellen kann, zum anderen wird Face von (statischen) kognitiven Grundlagen - wie zum Beispiel bestimmten Werten einer Gemeinschaft beeinflusst, gleichermaßen aber auch im Interaktionsvollzug (dynamisch) sozial konstituiert (vgl. Spencer-Oatey 2007: 654). Das bedeutet, dass Face eine gemeinsame Leistung ist, die unweigerlich die Verwendung von Sprache erfordert, da es als ein wesentlicher Bestandteil der Interaktion zwischen den Beteiligten hervorgebracht wird (vgl. Arundale 2010: 2079). So wie für Spencer-Oatey (2007) spielt auch für Arundale (2010: 2086) der Aspekt der Beziehung eines Individuums zu anderen Personen eine zentrale Rolle, da Face nicht auf der Ebene des Individuums als unabhängiges System konstituiert wird, sondern auf der Ebene eines minimalen sozialen Systems, das mindestens zwei interagierende Individuen umfasst (vgl. Arundale 2010: 2086). In diesem sozialen System beziehungsweise in der Interaktion mit anderen Individuen dient Face dazu, eine Verbindung mit oder eine Trennung von anderen Personen $\mathrm{zu}$ erreichen (vgl. Arundale 2010: 2086).

Für die Analyse im empirischen Teil dieser Arbeit bleibt festzuhalten, dass Face in der sozialen Interaktion eine zentrale Rolle spielt und dass dessen Hervorbringung eine gemeinsame Angelegenheit aller Beteiligten ist (vgl. Lerner 1996: 303), für die ihnen verschiedene Praktiken und Ressourcen zur Verfügung stehen. Gerade im Hinblick auf die Tatsache, dass die Gespräche ein geselliges Ereignis darstellen, in dessen Rahmen auch Kunstkommunikation betrieben wird und es besonders im Zusammenhang mit Bewertungen - zu Nichtübereinstimmung und 
damit verbunden auch zu offener Kritik und einer heiklen Situation kommen kann, werden Fragen des Face sowie Face-Work relevant (vgl. auch Schwitalla 1996: 285). Inwiefern die an den Gesprächen in der Theaterpause Beteiligten im Zuge ihrer Bewertungsinteraktionen auf Face-Work-Strategien zurückgreifen, Übereinstimmung anstreben und Nichtübereinstimmung $\mathrm{zu}$ vermeiden versuchen (vgl. Brown/Levinson 1987) sowie in der Interaktion Face-Work betreiben, um die Verbindung mit anderen zu stärken oder eine Trennung von anderen (Distinktion) zu forcieren (vgl. Arundale 2010), soll im empirischen Teil der vorliegenden Arbeit auf Basis der Gesprächsdaten herausgestellt werden.

\subsection{Zwischenfazit}

Die in den 1980er Jahren modellierten und in Kapitel 2.1 referierten handlungstheoretischen Ansätze zur Beschreibung von Bewertungen stellen zunächst eine hilfreiche erste Vororientierung und einen Ausgangspunkt für die Beantwortung der Frage dar, wann von einer Bewertung gesprochen werden kann. Aus konversationsanalytischer Perspektive sind sie aber aufgrund ihrer „Konzeptionen von ,Handlung‘, die die bewusste, zweckrationale Intentionalität des individuellen Akteurs zum grundlegenden Modell allen Handelns machen“ (Deppermann/Feilke/Linke 2016: 16; vgl. auch Habscheid 2016: 127) und aufgrund der Vernachlässigung sprachlicher (und multimodaler) Oberflächen, die den Kernpunkt einer linguistischen Rekonstruktion von Kommunikation ausmachen, längst nicht ausreichend, um der Komplexität sprachlichen Handelns und der Komplexität von Interaktionen, wie sie etwa in dieser Arbeit untersucht werden, gerecht zu werden (vgl. auch Feilke/Linke 2009; Habscheid 2011; für den Bereich der Textkommunikation vgl. Hausendorf et al. 2017). Dies gilt gleichermaßen für die Betrachtung von Handlungen als isolierte Einzelphänomene in einem hypothetischen „Null-Kontext“ (vgl. Sidnell/Enfield 2017 sowie Drew/ Heritage 1992: 13) und das damit einhergehende Ausblenden des Kontexts als für die Interagierenden relevante Intepretationsressource (siehe auch Kapitel 4.2).

Das Vernachlässigen des interaktiven Charakters der (kommunikativen) Bewältigung von Alltagsangelegenheiten ist ein Kritikpunkt, der manchen Handungstheorien aus einer praxeologischen, am praktischen Vollzug von Handlungen orientierten Perspektive zum Vorwurf gemacht wird (vgl. SchulzSchaeffer 2010: 322): „Die Handlungstheorie“, so Schulz-Schaeffer (2010: 320), „betrachte die sinnhafte Orientierung der Akteure als das Resultat eines bewusst intentionalen Prozesses“, der von Beteiligten bei der Realisierung von Alltagsangelegenheiten sprachlich aber selten bis gar nicht expliziert wird (vgl. unter anderem Deppermann/Feilke/Linke 2016; Fiehler 1990; Garfinkel 
1960/2012). Obwohl zu hinterfragen ist, inwieweit handlungstheoretische Ansätze generalisierend als „die Handlungstheorie“ $\mathrm{zu}$ fassen sind, ${ }^{29}$ so ist der Zweifel daran, dass dem Handeln der Akteure eine ihnen stets bewusste zweckrationale Intentionalität zugrunde liegt, berechtigt (siehe Kapitel 2.1 und 2.2). Garfinkels Annahme (siehe Kapitel 2.1.2), dass das Handeln der Akteure nur zu einem kleinen Teil ein bewusst zweckrationales Handeln ist, dem sie zu einem großen Teil einen nicht hinterfragten und „unbezweifelbaren Hintergrund von Dingen“ (Garfinkel 1960/2012: 57) zugrunde legen - das heißt ihr Handeln nicht stets bewusst reflektiert abwägen - bietet eine nachvollziehbare Perspektive, aus der beobachtet und rekonstruiert werden kann, in welchem Maße und auf welche Weise die Beteiligten in ihrem Handeln die diesem stillschweigend zugrunde gelegten Gegebenheiten (überhaupt) relevant setzen.

Eine solche an der Praxis der Akteure orientierte Perspektive bieten einige aus der Soziologie stammende und inzwischen auch in der Linguistik rezipierte praxeologische Ansätze. An der kommunikativen Praxis der Beteiligten orientierte Praktikenkonzepte, wie zum Beispiel das in Kapitel 2.2 vorgestellte Konzept der Co-Operative Action von Goodwin (2018), ermöglichen es, der vorangehend angesprochenen Komplexität, Kontingenz, Kontextbezogenheit, Dynamik und Interaktionalität sprachlichen Handelns gerecht zu werden und die wechselseitige Verfertigung (vgl. Schüttpelz/Meyer 2017) dieses sprachlichen Handelns adäquat rekonstruieren zu können, ohne dabei den kulturellen Hintergrund, in den die von den Beteiligten hervorgebrachten Praktiken eingebettet sind, zu vernachlässigen.

Wie das im Fokus der vorliegenden Arbeit stehende Bewerten im praktischen Vollzug von den Beteiligten interaktiv realisiert wird, wie damit einhergehend von den Beteiligten Wissen indiziert wird, soziale Positionierungen vorgenommen werden, andere Beteiligte zu Stellungnahmen verpflichtet werden können und wie die Beteiligten mit einer potenziellen Face-Bedrohung umgehen, zeigen konversationsanalytische Studien zu Bewertungsinteraktionen in privaten Alltagsgesprächen und institutionellen Zusammenhängen. Die in Untersuchungen zu Bewertungsinteraktionen in privaten Alltagsgesprächen teilweise konstatierte Allgemeingültigkeit konversationeller Prinzipien (zum Beispiel das Vorliegen einer Präferenz für übereinstimmende Zweitbewertungen; vgl. unter anderem Pomerantz 1984; Auer/Uhmann 1982), kann und muss auf Basis von Ergebnissen, die aus Untersuchungen institutioneller Gespräche gewonnen wurden, revidiert werden. Auf audiovisuellen Gesprächsdaten basierende Analysen

29 Vergleiche Holly, Kühn und Püschel (1984) zu einer Auseinandersetzung mit dem Handlungsbegriff in der linguistischen Pragmatik. 
zeigen beispielsweise, dass Eskalierungen und Deskalierungen situations- und rollenspezifisch vorgenommen werden (vgl. Lindström/Heinemann 2009 für Bewertungsinteraktionen in der häuslichen Altenpflege), dass Erstbewertungen nicht notwendigerweise mit Zweitbewertungen quittiert werden (vgl. Mondada 2009 für Gespräche zwischen Autoverkäufern und Kunden), sowie dass Nichtübereinstimmungen bei Bewertungen im Rahmen von gemeinsamen Entscheidungsprozessen einer Verbalisierung und eines Abgleichs von Gründen bedürfen (vgl. Fasulo/Monzoni 2009 für Gespräche in einem Modeatelier).

Die oben genannten Arbeiten bieten mit dem Herausstellen sowohl allgemeiner als auch institutionsspezifischer Ausprägungen von Bewertungsinteraktionen einen sinnvollen Ausgangs- und Anknüpfpunkt sowie eine Vergleichsgröße für die im empirischen Teil dieser Arbeit analysierten Bewertungsinteraktionen in Gesprächen in der Theaterpause. Aufbauend auf den in Kapitel 2 gewonnenen Erkenntnissen gilt es für die in Kapitel 8 dieser Arbeit analysierten Interaktionen - orientiert an der in den Gesprächen abgebildeten kommunikativen Praxis der Beteiligten - zu klären, inwieweit sich die in den in Kapitel 2.1 dargestellten rationalistisch-handlungstheoretischen Modellen herausgestellten Bewertungscharakteristika in den Theaterpausengesprächen manifestieren beziehungsweise von den Beteiligten überhaupt offen gelegt werden. Des Weiteren gilt es, die mit Bezug auf die in der konversationsanalytischen Forschung zum Bewerten in privaten Alltagsinteraktionen herausgestellten Prinzipien (siehe Kapitel 2.2) mit Blick auf den semi-institutionellen Kontext der Theaterpausengespräche auf ihre Gültigkeit für diesen Gesprächstyp zu prüfen. 